\title{
The enigmatic binary system HD 5980
}

\author{
D. John Hillier ${ }^{1 \star}$, Gloria Koenigsberger², Yaël Nazé3, \\ Nidia Morrell ${ }^{4}$, Rodolfo H. Barbá ${ }^{5}$, Roberto Gamen ${ }^{6}$ \\ 1 Department of Physics and Astronomy \&S Pittsburgh Particle Physics, Astrophysics and Cosmology Center (PITT PACC), \\ University of Pittsburgh, 3941 O'Hara Street, Pittsburgh, PA 15260, USA \\ 2 Instituto de Ciencias Físicas, Universidad Nacional Autónoma de México, Ave. Universidad S/N, Cuernavaca, Morelos, \\ 62210, México,gloria@astro.unam.mx \\ 3 FNRS/STAR Institute, University of Liège, Alleée du 6 Août 19c, B5C,B4000-Liège, Belgium, ynaze@uliege.be \\ 4 Las Campanas Observatory, The Carnegie Observatories, Colina El Pino s/n, Casillas 601, La Serena, Chile, nmorrell@lco.cl \\ 5 Departamento de Física y Astronomía, Av. Juan Cisternas 1200 Norte, Universidad de la Serena, La Serena, Chile, rbarba@userena.cl \\ ${ }^{6}$ Facultad de Ciencias Astronómicas y Geofísicas, Universidad Nacional de La Plata, and Instituto de Astrofísica de La Plata \\ CCT La Plata, CONICET-UNLP, Paseo del Bosque S/N, B1900FWA, La Plata, Argentina, rgamen@fcaglp.unlp.edu.ar
}

Accepted XXX. Received YYY; in original form ZZZ

\begin{abstract}
The Small Magellanic Cloud multiple system HD 5980 contains a luminous blue variable (LBV) that underwent a major eruption in 1994, and whose current spectrum is that of a hydrogen-rich Wolf-Rayet (WR) star. Since the eruption, the wind mass-loss rate has been declining while wind speeds have been steadily increasing. Observations obtained in 2014 when Star A (the LBV) eclipses Star $B$ indicate that the fitted mass-loss rate and luminosity have reached the lowest values ever determined for such spectra: $\dot{M}=4.5 \times 10^{-5} M_{\odot} \mathrm{yr}^{-1}, L=1.8 \times 10^{6} L_{\odot}$. In addition, the radius of the LBV's continuum-emitting region is similar to that derived from the eclipse light curves of the late 1970s. Hence, it appears to have attained a similar "low" state to that of the late 1970s. While a good fit to the emission spectrum is obtained using a CMFGEN model, there are discrepancies in the UV. In particular, the extent of the observed absorption profiles is $\sim 1000 \mathrm{~km} \mathrm{~s}^{-1}$ greater than predicted by the emission-line intensities. Further, HST UV observations obtained in 2016, when Star A is eclipsed by Star B, show unusual P Cygni profiles that are not easily explained. Surprisingly the 2016 emission line spectrum is similar to that at the opposite eclipse obtained in 2014. The complex UV profiles are likely to arise as a consequence of the dynamics of the wind-wind collision and radiative braking, both of which will cause significant departures from spherical symmetry, and have a strong orbital phase dependence. However, other scenarios, such as intrinsically aspherical winds, cannot be ruled out.
\end{abstract}

Key words: stars: binaries: eclipsing; stars: individual(HD 5980); stars: variables: luminous blue variables; stars: Wolf-Rayet

\section{INTRODUCTION}

HD 5980 is a multiple system in the Small Magellanic Cloud $(\mathrm{SMC})$ containing a massive star $\left(\mathrm{M} \sim 61 \pm 10 M_{\odot}\right.$; Koenigsberger et al. 2014), referred to as Star A, which underwent a luminous blue variable (LBV) type eruptive event during 1993-1994. Its current spectrum is that of a WolfRayet (WR) nitrogen-sequence WN5-6. It has a close eclipsing companion that is believed to also be a WR star of similar spectral characteristics which is named as Star B (M $\sim 66 \pm 10 M_{\odot} ;$ Koenigsberger et al. 2014). These two stars cycle around each other with a $P_{A B}=19.3 \mathrm{~d}$ and an eccentricity of 0.3 . A third light source is also present in spectra of HD 5980. This object, called Star C, possesses a late-O-

^ E-mail: hillier@pitt.edu (DJH) type spectrum and is believed to be a supergiant. Its photospheric absorption lines undergo periodic radial velocity shifts indicative of a $P_{C}=97 \mathrm{~d}$ and $e_{C}=0.8$ orbit. With such a large eccentricity and short period, Star $C$ cannot be in orbit around the $A B$-pair according to the stability criteria of Mardling \& Aarseth (1999) as can be seen from Eq. (23) in Toonen et al. (2016). Hence, Star C is itself a binary system which may or may not be in a very long period orbit around the $\mathrm{AB}$ pair.

The potential importance of the HD 5980 system for understanding LBVs, stellar evolution and stellar winds cannot be understated. For example, due to its large masses and the low metallicity of the SMC, the system could evolve into a black hole - black hole binary, and hence it could be similar to the progenitor systems that gave rise to black hole mergers detected by LIGO (Abbott et al. 2016). The similarity 
of the two WN stars, and the short orbital period, indicate that their winds will undergo complex interactions and will be influenced by the radiation field of the companion star. Moreover the variability of Star A indicates that the nature and strength of these interactions will change with time. Due to its importance we have been obtaining spectra of the system on a regular basis. Observation of P Cygni lines in the UV are of particular importance, since the absorption components in such lines trace gas along the line of sight.

$\mathrm{P}$ Cygni spectral lines are widely used to infer the wind speed, mass-loss rates and ionization state of the winds of O-type stars, WR stars, and LBVs. The method by which these parameters are determined consists of fitting theoretical model line profiles and spectral energy distributions to the observations. With few exceptions, the theoretical models assume that the stellar wind consists of a spherically symmetric, monotonically accelerating outflow and that the central source irradiates the wind in a similarly symmetrical manner. Furthermore, the models assume that the wind is in a stationary state or that, at worst, its variability does not strongly affect its large-scale structure.

In binary star systems the situation is much more complex. For example, there will be a wind-wind collision that will produce shocks, X-rays, density enhancements, and inhomogeneities. Further, the radiation field of one star can modify the wind structure of the second star, and if the orbits are elliptical, the changes will depend on orbital phase.

Despite its binary nature, theoretical models have been quite successful in fitting the observed spectra of HD 5980 during epochs in which the erupting star's wind dominated, suggesting that the departures from the simplifying assumptions did not strongly influence the spectrum. However, the new UV spectra that we report in this paper indicate that asymmetries (e.g, binary interaction via winds and the radiation fields) cannot be neglected, and reveal that there is much in this system that we do not understand.

In this paper we are primarily concerned with HST data obtained in 2014 and 2016, and that are described in Section 2. An historical overview of the HD 5980 system is provided in Section 3. In Section 4 we discuss the binary system in detail, laying the groundwork for understanding our new (and future) observations. In this section we discuss stellar winds (§4.1), eclipses (§4.2), short-term photometric variability (§4.3), Star B (§4.4) and Star $C(\S 4.5)$. A discussion of spectral variations and further inference about Star $A$ and Star $B$ is provided in Section 5. The results of performing CMFGEN fits to the 2014 observations are described in Section 6, while in Section 7 we discuss the extended absorptions seen on the UV resonance transitions ( $\S 7.1$ ), and the unusual profiles (particularly the C IV resonance transition) obtained in 2016 (§ 7.2).

\section{NEW HST OBSERVATIONS}

The HST/STIS observations of HD 5980 were obtained under Program ID 13373 on 2014 March 24 and under Program ID 14476 on 2016 September 21. Both sets of observations, summarized in Table 1, were acquired with the same setup. Specifically, two medium dispersion UV spectra were obtained with the FUV MAMA, E140M grating (1100-1700 ^) through the $0.2 \times 0.2$ arcsec slit. These were followed in rapid succession by three low dispersion CCD spectra covering the wavelength range 1700-10 $000 \AA$.

Based on the Sterken \& Breysacher (1997) ephemeris for HD 5980, the 2014 spectra were obtained at orbital phase $0.004 \pm 0.001$ which corresponds to the (partial) eclipse in which Star A passes in front of Star B. The opposite (partial) eclipse occurs at $\phi=0.36$. The 2016 observations were obtained at $0.349 \pm 0.002$.

Foellmi et al. (2008) suggested a possible correction in the initial epoch for the orbital phase calculation (new value $\left.T_{0}=2443158.865\right)$ which they needed to align the $\phi=0.36$ minimum in the 2003 and 2006 photometric data with that of 1978. If the need for this shift is confirmed, then our 2014 observations would have been obtained at $\phi=0.995 \pm 0.001$, at which time any $\mathrm{P}$ Cygni absorption components arising in Star B would still be eclipsed. The 2016 observations would have been obtained within the phase range 0.338-0.344. Although dependent on the current size of the optically-thick region in Star $A$, it is unlikely that Star $A$ was totally eclipsed $^{1}$. For consistency with our previous papers, and until an ephemeris modification is confirmed, we will continue to use the Sterken \& Breysacher (1997) ephemeris.

The data were processed with the standard STIS pipeline calibration ${ }^{2}$. In order to ensure homogeneity, we retrieved our previous STIS observations ${ }^{3}$ obtained in 1999, 2000, 2001 and 2009 from $\mathrm{MAST}^{4}$, all of which were reprocessed with the same STIS calibration pipeline as used for the 2014 and 2016 observations.

\section{HISTORICAL PERSPECTIVES}

As our understanding of HD 5980 is far from complete, and since the historical record may influence our interpretation of the current observations, we provide here a brief historical perspective of the system.

\subsection{Photometric history}

The Harvard Patrol Plates covering epochs between 1899 and 1951 do not reveal large visual magnitude fluctuations (M. Hazen 2005, private communication cited in Koenigsberger et al. 2010), although they do indicate that $\mathrm{B} \sim 11.4$ until around 1918, when a slow decline to $\mathrm{B} \sim 11.8$ occurred. Between 1936 and 1951 it remained at this low state, but with several brief brightness enhancements. Photometric measurements during 1970 to 1980 indicate $\mathrm{V} \sim 11.86$ (Azzopardi \& Vigneau 1975) to V 11.6 (Feast et al. 1960). The long term continuum variability after 1979 can be described in terms of two distinct characteristics. The first is

1 The STIS/MAMA spectrum of 2014 was obtained in 2 successive acquisitions and the second one is at slightly lower flux levels than the first one. According to the modified $T_{0}$, the corresponding orbital phases would have been 0.993 and 0.997 , respectively, consistent with a deeper eclipse during the second segment.

2 http://www.stsci.edu/hst/stis/documents/handbooks/currentIHB/stis_ihb.pdf

3 Data sets O55Q, O6CD, OB2N

4 STScI MAST: Mikulski Archive for Space Telescopes. STScI is operated by the Association of Universities for Research in Astronomy, Inc., under NASA contract NAS5-26555 
Table 1. STIS 2014 and 2016 observations of HD 5980. We list the identifier of the data set in the first column, the grating that was used in the second, the resolving power in the third, the exposure time in the fourth, the wavelength coverage in the fifth and in the sixth column the approximate signal-to-noise ratio (SNR) at the wavelength listed in the seventh column. $R$ is calculated as $\lambda / 2 \Delta \lambda$ where $\Delta \lambda=0.05 \AA$ per pixel (STIS Instrument Handbook, Table 4.1).

\begin{tabular}{llcrlll}
\hline Set ID & Grating & $R$ & $\begin{array}{r}t_{\exp } \\
(\mathrm{sec})\end{array}$ & $\begin{array}{l}\lambda \text {-range } \\
(\AA)\end{array}$ & $S / N$ & $\begin{array}{l}\lambda \\
(\AA)\end{array}$ \\
\hline \hline ocax0 & 2014 & & & & & \\
1010 & E140M & $11400-17400$ & 2608 & $1150-1700$ & 25 & 1525 \\
1020 & E140M & $11400-17400$ & 2070 & $1150-1700$ & 20 & 1525 \\
1030 & G230LB & $620-1130$ & 300 & $1700-3050$ & 60 & 2100 \\
1040 & G430L & $530-1040$ & 60 & $2900-5670$ & 70 & 3600 \\
1050 & G750L & $530-1040$ & 60 & $5300-10200$ & 70 & 5680 \\
od3g0 & 2016 & & & & & \\
1010 & E140M & $11400-17400$ & 2837 & $1150-1700$ & 27 & 1525 \\
1020 & E140M & $11400-17400$ & 2028 & $1150-1700$ & 21 & 1525 \\
1030 & G230LB & $620-1130$ & 300 & $1700-3050$ & 43 & 2100 \\
1040 & G430L & $530-1040$ & 60 & $2900-5670$ & 30 & 3600 \\
1050 & G750L & $530-1040$ & 60 & $5300-10200$ & 38 & 5680 \\
\hline
\end{tabular}

a slow increase in visual brightness which started in $\sim 1982$ peaked in 2000, and then has been declining to the present day. The second consists of two sudden and relatively brief episodes of brightness enhancements, the first in $1993(\mathrm{~V} \simeq$ 10) and the second in $1994(\mathrm{~V} \simeq$ 8.7) (Bateson \& Jones 1994). The latter had a duration $\leq 1 \mathrm{yr}$, approximately a factor of 4 longer than the first event (Koenigsberger 2004; Bateson \& Jones 1994).

\subsection{Overview of optical spectral variations}

Photographic plates obtained in 1955-1965 in the South African Astronomical Observatory were digitized and analyzed in Koenigsberger et al. (2010). The spectra of 19561962 display strong and broad He II $\lambda 4686$, as well as $\mathrm{H} \beta+\mathrm{He}$ II, He II $\lambda 4542, \mathrm{H} \gamma+\mathrm{HeII}$ emission, and on most spectra, N IV $\lambda 4058$. This N IV line lies near the end of the tracings where the noise generally increases significantly. However, it is absent in a few spectra with relatively good S/N, particularly starting in 1962 .

Walborn (1977) described his visual spectrum of 1973 and 1977 as follows: "broad He II $\lambda 4686$ emission with extensive wings; P Cygni profiles at N V $\lambda \lambda 4604,4620$; strong N IV $\lambda \lambda 3479$ - 3485 emission but no N IV $\lambda 4058$. Wellmarked He I absorption, but only traces of He II". This led him to classify it as OB?+WN3. Hence, the apparent absence of N IV $\lambda 4058$ seen in the 1962-1965 photographic plates seems to be real. The earliest spectra indicate that the WR component had a WN4 type spectrum, which gradually evolved to WN6 in 1991 (Koenigsberger et al. 1994). By 1993 this spectrum was WN7. The 1993 event is associated with a shift in spectral type from WN6/7 to WN8 and back again (Moffat et al. 1998). The more violent 1994 event involves an even greater shift in the dominant spectral type.

A striking feature of the early (1955-1965) spectra is the variability in the width of the emission lines, first reported by Feast et al. (1960). The full width at half maximum (fwhm) intensity of He II $\lambda 4686$, for example, has values ranging from $1000 \mathrm{~km} \mathrm{~s}^{-1}$ to $2200 \mathrm{~km} \mathrm{~s}^{-1}$ (Koenigsberger et al.
2010). In the few spectra with good enough $\mathrm{S} / \mathrm{N}$ at $\mathrm{N}$ IV $\lambda 4058$ and in which it is present, this line seems to behave similarly. Breysacher \& Perrier (1980) pointed out the orbital-phase dependence in the fwhm variations: lines are narrowest during conjunctions compared to other phases, with maxima occurring around elongations, and an even more remarkable feature is that this variability persists throughout all epochs during which spectra are available (Koenigsberger et al. 2010; Moffat et al. 1998). Moffat et al. (1998) point out the uniqueness of this type of variability. Indeed, to our knowledge, it is not observed in any other WR binary system.

Due to the similarity in magnitudes of Star $A$ and Star B, the precise spectral classes of the two components are unknown. In the unnormalized spectra illustrated by Breysacher \& Westerlund (1978) absorption in higher members of the Balmer series is clearly evident, and this absorption was associated with one of the stars in the system. However, given the visual magnitude of the system at that time, and the strengths of higher Balmer lines relative to the Ca II interstellar line, which are similar to that seen in today's spectra, the higher Balmer series members must be strongly affected by Star $C$.

The earliest optical spectrum of the 1994 eruption was obtained in September of that year, and shows strong $\mathrm{H}$ emission lines (Barbá et al. 1995; Heydari-Malayeri et al. 1997), indicating that the star undergoing the outburst contained hydrogen. In addition to the Balmer-H emission lines, the visual spectrum shows He I P Cygni lines, as well as low-ionization emission from N II, Si III, Fe III (Heydari-Malayeri et al. 1997). The optical spectrum was very similar to that of P Cygni (B1Ia), except for the presence of the weak and broad He II $\lambda 4686$ line. This high ionization line increased in strength by $\sim 50 \%$ between September 10 (phase 0.68) and September 12 (phase 0.72) and its radial velocity (RV) appears to have changed from 66 to 129 $\mathrm{km} \mathrm{s}^{-1}$. These speeds correspond to -84 and $-21 \mathrm{~km} \mathrm{~s}^{-1}$ in the HD 5980 rest frame, adopting a systemic speed of +150 $\mathrm{km} \mathrm{s}^{-1}$ for the SMC. Thus, if these RVs correspond to or- 


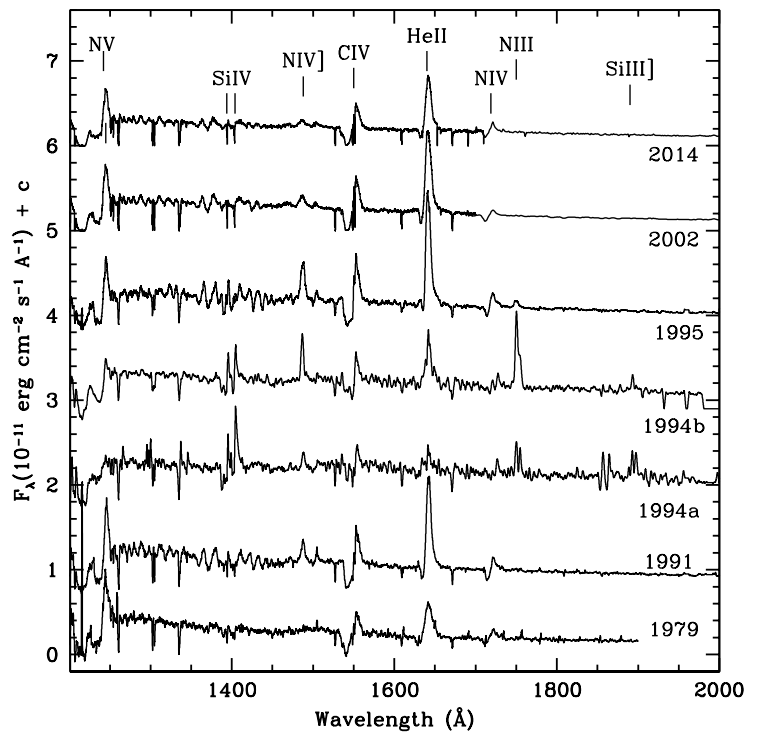

Figure 1. Montage of UV spectra from 1979 to 2014. Spectra are successively shifted vertically by $10^{-12} \mathrm{erg} \mathrm{cm}^{-2} \mathrm{~s}^{-1}$ for clarity in the figure. The spectra and corresponding orbital phases are plotted from bottom to top as follows: SWP4345 (0.91), SWP42470 (0.05), SWP52888 (0.51), SWP53226 (0.43), SWP56205 (0.99), o6cd01010+o6cd01020 (0.99), ocax01010+ocax1020 (0.99). Particularly striking in these low resolution plots is the distinct 1994outburst spectrum, and the variation of the Si IV $\lambda \lambda 1394,1403$ and N IV] $\lambda 1486$ lines. SWP refers to the short wavelength prime camera of IUE.

bital motion, then He II $\lambda 4686$ would have had its origin in Star A, not Star B as had been assumed.

In present spectra the He II Pickering decrement clearly shows an odd-even effect indicating that at least one star has H. However, it is unclear whether Star B contains H. In the galaxy, most WNE stars exhibit little or no $\mathrm{H}$. However, in the SMC most of the WN stars are of type WNE, they are relatively weak lined, show absorption lines, and generally contain hydrogen (Hainich et al. 2015).

\subsection{Overview of UV spectral variations}

The UV spectral characteristics of HD 5980 are illustrated by Figure 1, which shows the spectral evolution over the time frame 1979-2014. Detailed descriptions for UV data up to 2009 are given in Koenigsberger (2004), Koenigsberger et al. (1998a), Koenigsberger et al. (2010), Koenigsberger et al. (2014), and references therein.

The slow brightness increase from 1982 through 2000 correlates with an increase in emission-line strength, a decrease in width, and a corresponding decrease in the terminal wind speed, $\mathrm{V}_{\infty}$ (Georgiev et al. 2011). Outflow speeds are in the range $\sim 1700$ to $2200 \mathrm{~km} \mathrm{~s}^{-1}$. This trend is interrupted, however, during the 1994 eruption when the dominant lines now arise from lower ionization species such as Si II, Si III, Fe III, and Al III, most of which display P Cygni profiles indicating outflow speeds of $\sim 200$ to $600 \mathrm{~km} \mathrm{~s}^{-1}$. At this stage, the spectrum was shown to be very similar to that of the B1.5I ${ }^{+}$star HD 1822 by Koenigsberger et al. (1996), except for the abnormally strong and wide Si IV $\lambda \lambda 1394,1403$ lines.
In the earliest UV spectrum obtained in 1994 the expansion speed deduced from the P Cygni lines correlates with the degree of ionization: Si IV indicates $\mathrm{V}_{\infty} \simeq 1640 \mathrm{~km} \mathrm{~s}^{-1}$, $\mathrm{Al}$ III $\lambda 1854$ indicates $\mathrm{V}_{\infty} \simeq 670 \mathrm{~km} \mathrm{~s}^{-1}$ while the lower ionization lines indicate speeds $\leq 400 \mathrm{~km} \mathrm{~s}^{-1}$, the latter consistent with what is observed by Heydari-Malayeri et al. (1997) in the optical P Cygni lines.

By 1995 March, the UV spectrum has reverted to a typical WR spectrum, with P Cygni lines of N V, He II, C IV, Si IV and N IV, similar to its appearance in 1991 except for the stronger line intensities. This is one of the striking features of the post-eruption spectra: even though the spectral type reverts to that of pre-eruption states, the emission lines continue to increase in strength (equivalent width), reaching maximum in 1999-2000 (Koenigsberger et al. 2010). This behavior mimics that of the continuum intensity $\left(m_{v}\right)$ which also peaks around the year 2000, after which it starts to decline, as do the emission-line strengths.

Clear evidence for WR features together with the eruption spectrum are seen in data taken on 1994, December 31 (phase 0.43; labeled $1994 b$ in Fig. 1) - in particular N V $\lambda \lambda 1238,1242$, C IV $\lambda \lambda 1548,1552$, and He II $\lambda 1640$ are seen (Koenigsberger 2004), however they are heavily contaminated by the wind/ejecta of the outbursting star. At this epoch the sudden outburst phase is winding down - the visual flux is roughly 1 magnitude fainter than at peak. In UV data taken a month earlier, on 1994 November 24, at phase 0.5 , the evidence for UV WR features is less clear although the presence of He II $\lambda 1640$ and C IV $\lambda \lambda 1548,1552$ emission can be gleaned, especially by comparison with the 1994 December 31 spectrum. Moffat et al. (1998) note the abnormal strength of He II $\lambda 4686$ in spectra taken even earlier, in 1994 September (Barbá et al. 1995) and October (Heydari-Malayeri et al. 1997). It was the continued presence of WR features in the outburst spectrum, presumably arising from Star B, that suggested that it was Star A that was varying. The presence of a hydrogen contribution in the outburst spectrum reinforced this notion, since a bona fide WNE-type star, as Star B was believed to be, is not expected to have a significant amount of this element. As noted earlier, this argument is flawed. WNE stars can have hydrogen, and at the present time it appears that both Star A and Star B have similar WNE like spectra. Fortunately RV observations confirm that it was Star A that varied. Specifically, emission lines that were absent or very weak prior to the active phase - N IV] and the Fe VI in the UV - were shown to follow the orbital motion of Star A (e.g. Barbá et al. 1996). All subsequent analyses assume that only Star A varies.

The presence of WNE features in the 1994 SeptemberNovember spectra is somewhat surprising since in 1994 September the $\tau=2 / 3$ radius derived by Drissen et al. (2001) of $280 R_{\odot}$ exceeded the semi-major axis of the system by almost a factor of 2, and hence Star $B$ would have been completely engulfed by the wind of Star A. Even in 1994 December, when the $m_{v}$ was in decline, a radius of $130 R_{\odot}$ was inferred by Drissen et al. (2001). Given the wind parameters derived at that time $\left(\dot{M}=10^{-3} M_{\odot} \mathrm{yr}^{-1}, V_{\infty}=600 \mathrm{~km} \mathrm{~s}^{-1}\right)$, Star $B$ would still be engulfed by the wind of Star A. Two scenarios can account for this apparent contradiction: 1) Star $A$ developed WR emission lines very shortly after the 1994 maximum; 2) Star B altered the eruption wind structure in 


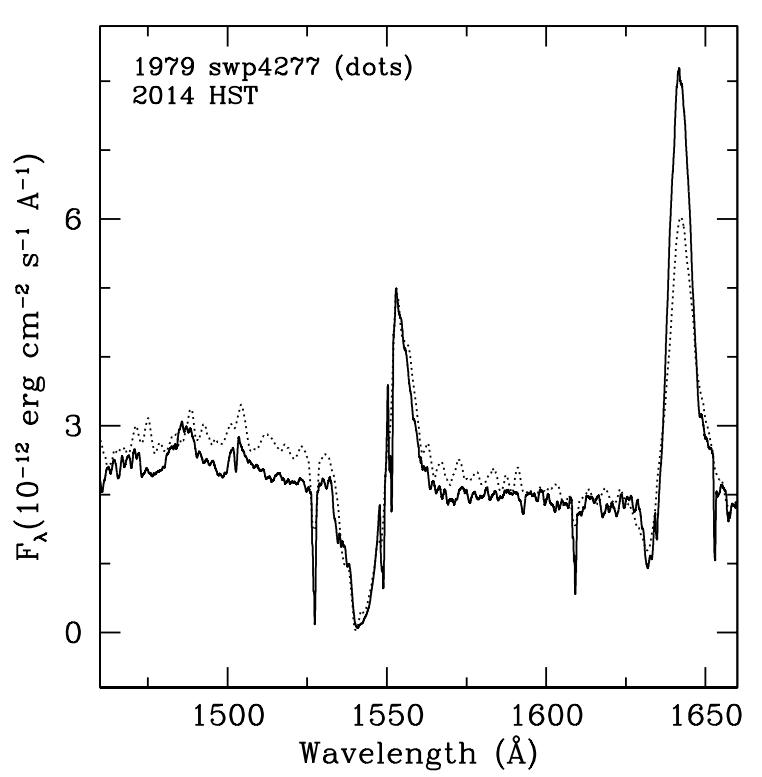

Figure 2. Comparison of UV spectra in 1979 (SWP 4277, $\phi=0.57$, dots $)$ and $2014(\phi=0.99)$ showing that the continuum and line fluxes of HD 5980 have declined to levels that are similar to those observed $\sim 35$ years ago. Further, the lines have similarly extended P Cygni absorption components. Flux is given in absolute flux units, not corrected for reddening.

its vicinity sufficiently so as to allow its spectrum to reach the observer.

Illustrated in Fig. 2 is the comparison of the C IV $\lambda \lambda 1548,1552$ and He II $\lambda 1640$ lines in 1979 and 2014, showing that the spectrum in 2014 has reverted to its pre-1980s characteristics.

\section{INSIGHTS INTO THE SYSTEM AND ITS WINDS}

\subsection{The winds}

To lay foundations for this and subsequent investigations, we briefly discuss the winds, line formation, and the relative scale of the winds and binary separation.

The orbital separation in HD 5980 is only 5 to 8 times larger than the stellar radii. Thus, the winds of both stars do not reach terminal velocity in the space between them. This statement is independent of the influence of radiative braking (Gayley et al. 1996). The classic wind velocity law is

$V(r)=V_{\infty}\left(1-R_{*} / r\right)^{\beta}$.

Adopting $20 R_{\odot}$ as a typical stellar radius, $\beta=1$, and a distance equal to the average separation between the stars (i.e., $r=a \sim 150 R_{\odot}$ ), the wind only reaches $87 \%$ of its terminal velocity in the direction of the companion. In reality, there will be a wind-wind collision between the stars. If we were to assume that the stars have equal wind momenta, then $V(\max )=0.73 V_{\infty}$. If $\beta$ is larger than 1 (as suggested by the analyses of Georgiev et al. 2011), the reduction in wind speed along the major axis may be even larger. This will be even more likely near periastron because of the non-negligible eccentricity. Further, as the evidence suggests that the stars have similar luminosities and effective temperatures, radiative braking must be playing a role.

As noted earlier, the orbital period of the $\mathrm{AB}$ system is 19.3 days (Breysacher \& Perrier 1980). Using a separation $r=a \sim 150 R_{\odot}$ (i.e., $r \sim 7 R_{*}$ ) and $V=2000 \mathrm{~km} \mathrm{~s}^{-1}$ in the above expression, the typical flow time is 0.60 days. This is a non-negligible fraction of the orbital period, especially when we note that significant changes in spectra are seen on this timescale (see 4.4), and since the resonance transitions can be optically thick beyond 100 stellar radii (corresponding to a flow time of $\sim 8$ days).

\subsection{The eclipses}

Analysis of photometric data obtained prior to 1991 (and hence prior to the 1994 outburst) reported by Perrier et al. (2009) indicates that Star A contributes $\sim 40 \%$ to the $V$ band light, Star B 30\%, and Star C 30\%. Neither of the eclipses is total - the analysis of Perrier et al. (2009) suggests that during the primary eclipse (i.e., the eclipse of Star $B$ ) only $\sim 70 \%$ of Star B's light is blocked, while during the secondary eclipse only $\sim 55 \%$ of Star $A$ 's continuum light is blocked. In their analysis Perrier et al. (2009) allowed for an extended atmosphere around Star B but assumed that Star $A$ was an OB star without an extended atmosphere.

The depth of the eclipses will be wavelength dependent due to the wavelength dependence of bound-free and freefree processes, and the presence of Star C. In particular, we would expect the eclipses in the near IR (e.g., at $2 \mu \mathrm{m}$ ) to be different from those in the optical and UV because free-free processes are more important, and because the influence of Star $C$ on the light curve will be less - in the V band Star $A$ is only 30 to $40 \%$ brighter than Star $C$ but at $2 \mu \mathrm{m}$ it is over three times brighter. ${ }^{5}$ Additionally, the eclipses will be much deeper in the continuum than in emission lines, and because emission lines are formed at different locations, the eclipse depth will vary between emission lines.

If we consider spectra obtained in 1986 with the International Ultraviolet Explorer (IUE), it can be shown that after correcting for the presence of Star $C$ by subtracting the spectrum of the supergiant Sk 80 , the spectrum at $\mathrm{phi}=0.35$ is very similar to that of phi $=0.51$ multiplied by 0.40 . This implies that $60 \%$ of the continuum was eclipsed in 1986 , consistent with the $55 \%$ stated above. Also, as expected, a smaller fraction of the emission lines was eclipsed.

As a simple demonstration of eclipse effects we take a model for Star A and compute the spectrum originating outside an impact parameter of $25 R_{\odot}$. This simulates the spectrum that would be observed if Star $B$ were a simple occulting disc located far from Star A. In general, the spectrum is very similar to the unocculted star, although, as to be expected, the equivalent width (EW) of emission lines has increased and there is a weakening of the $\mathrm{P}$ Cygni absorption components (Figs. $3 \& 4$ ). One distinct difference occurs in the UV - in the occulted spectrum the Fe forest is noticeably

5 Tests indicate the light curves in the K band may actually be similar to those in the optical - an unfortunate consequence arising from the weakening contamination of Star $C$ being offset by the extended photospheres of the two WR stars. 

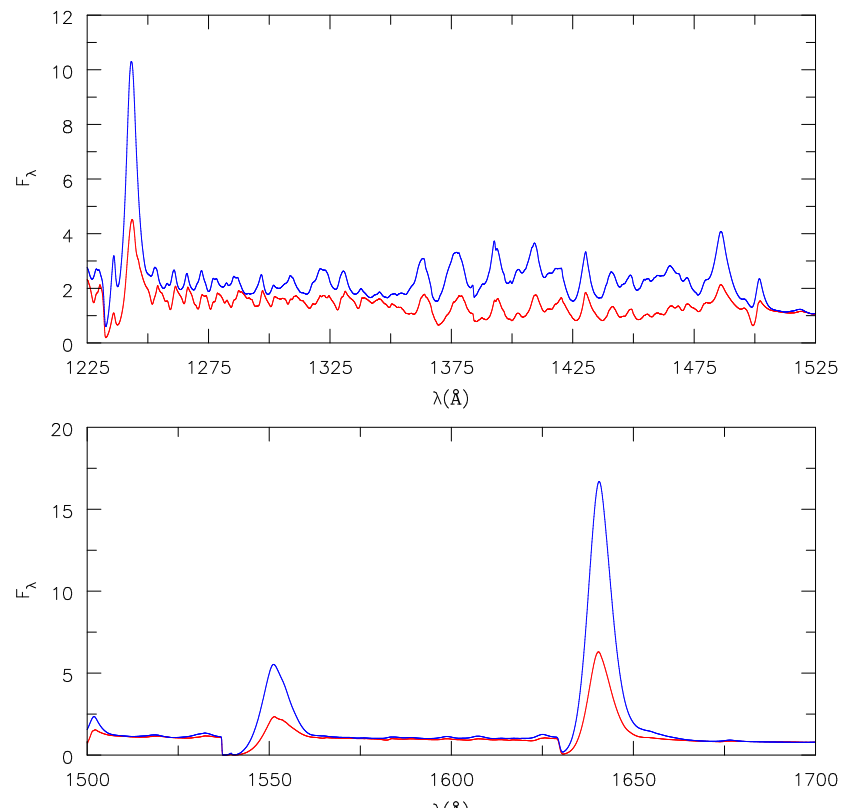

$\lambda(\AA)$

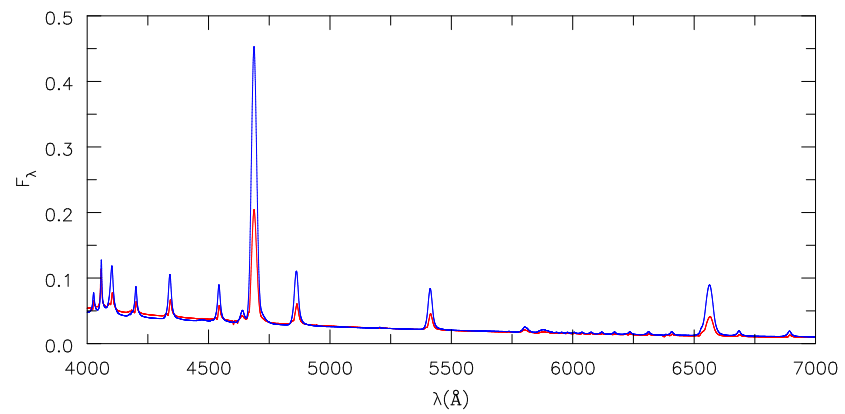

Figure 3. In red we show the spectrum of Star $A$ while in blue we show the spectrum that would be obtained if Star $A$ were occulted by a simple disk with radius $25.1 R_{\odot}$. To facilitate the comparison of line profiles, we have scaled the occulted spectrum by a factor of 3.17 in the UV, and 2.50 in the optical. Because the line emitting region is larger, the lines suffer less blocking than the continuum.

weaker. To match the V-band light curves of Perrier et al. $(2009)^{6}$, about $40 \%$ of Star A's light is not blocked when it is eclipsed by Star B. In reality the eclipse spectra are more complicated than this - the stars are located in each other's winds, there is a wind-wind interaction, and along our sightline, the line photons emitted by the more distant star can be resonantly absorbed/scattered by the second wind.

In 2000 Star $A$ was roughly $60 \%$ brighter than in 2014 in the optical and $\sim 20 \%$ in the UV, and thus the influence of Star B at phase 0.0 should be smaller than that inferred from analyses of the pre-outburst light curve. However, with the fading of Star A, Star B will have a more significant influence. According to the light curve of Perrier et al. (2009), when Star A eclipses Star B, about $20 \%$ of the observed Vband continuum, corrected for Star $C$, arises from Star B. However, as noted earlier, the influence on the emission lines will be larger.

${ }^{6}$ Concurrent light curves are unavailable. Given the variability of the system, these need to be obtained on a regular basis.
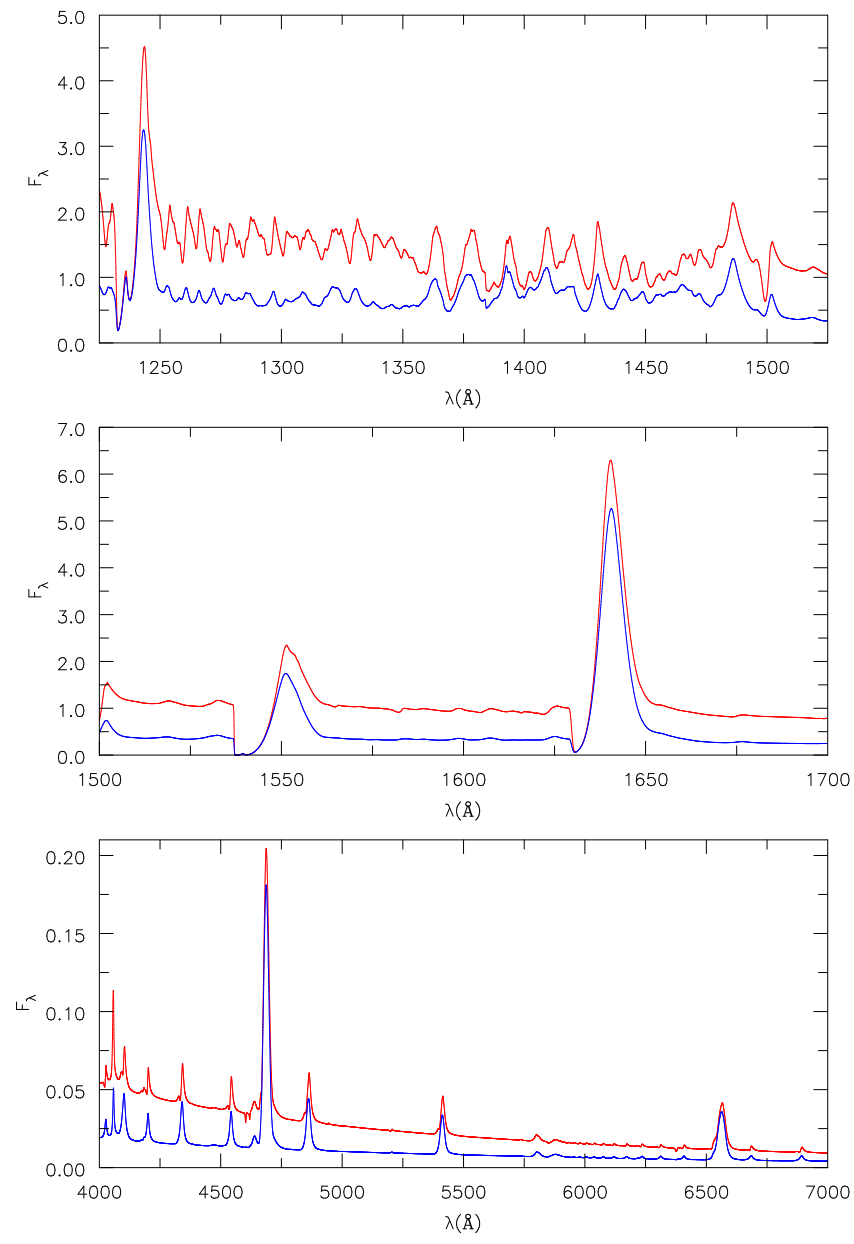

Figure 4. In red we show the spectrum of Star $A$ while in blue we show the spectrum that would be obtained if Star $A$ were occulted by a simple disk with radius $25.1 R_{\odot}$. No scaling is done so that fluxes can be compared. Because the line emitting region is larger, the lines suffer less blocking than the continuum. However, the strong resonance lines still exhibit their "classic" P Cygni profiles.

\subsection{Short-term variability}

Photometric data indicate that HD 5980 exhibits microvariations not directly related to its orbital period. Breysacher (1997) and Sterken \& Breysacher (1997) find a period of $\sim 6 \mathrm{hrs}$ and an amplitude of $0.025 \mathrm{mag}$ in data obtained shortly after the 1994 eruption and suggest that it might be due to pulsations in one of the two stars. As the oscillations persist through the eclipses, an alternative possibility is that they are associated with Star C. Although most $\mathrm{O}$ stars do not exhibit such variations, tidally excited oscillations (TEOs) have recently been reported in the OBtype system $\iota$ Orionis (Pablo et al. 2017) and $\eta$ Carinae (Richardson et al. 2018).

\subsection{Star B}

Observations before and after the eruption suggest that Star B is also a Wolf-Rayet star (e.g. Niemela et al. 1997). In early literature there is considerable confusion regarding the spectral types of Star $A$ and Star $B$ because of 
the presence of absorption lines from Star C. Its existence was not recognized until a RV study by Niemela (1988), and it was later confirmed via spectroscopic observations (Schweickhardt 2000; Schweickhardt et al. 2000; Koenigsberger et al. 2002) and photometric observations (Breysacher \& Perrier 1991; Perrier et al. 2009). While it is obvious that Star A is variable, it is unclear whether Star B is also an intrinsic variable ${ }^{7}$. Both stars exhibit emission in N V $\lambda \lambda 4604,4620$, although it appears to be stronger in Star $A$ (Foellmi et al. 2008; Koenigsberger et al. 2014). Extensive observations presented by Koenigsberger et al. (2014) show that the N V $\lambda 4944$ emission is double peaked during elongations and single peaked during eclipses, and that the corresponding RVs track the orbital motion of each star. It is the RV variation of the N V $\lambda 4944$ line that has been used to infer the stellar masses. Unfortunately the $\mathrm{N} V$ lines do not, by themselves, indicate the type of WR star. It could have a fairly intense emission line spectra (with emission in all of the He II Pickering lines, etc., similar to a strong-lined WNE star) or it could have a spectrum more closely resembling a weak-lined hot WR star with upper Balmer and He II (n-4) transitions only weakly in emission, or even in absorption (Hainich et al. 2015; Neugent et al. 2017).

In 2005 , the $\mathrm{N}$ IV $\lambda 4058$ transition was primarily due to Star $A$ - this is apparent from its radial velocity variation, and the relatively small changes in profile shape. The association of N IV with Star A is consistent with inferences from UV spectra - the N IV] $\lambda 1486$ line is weakening with time confirming that it was Star A that had the outburst in 1994. The association of N IV $\lambda 4058$ with Star A is somewhat surprising as observations (during the period 1980 to 1983) by Niemela et al. (1997) showed that its radial velocity tracked that of Star B.

In the analysis presented by Koenigsberger et al. (2014) it was assumed that in 2009 Star B presented the same spectrum as Star $A$, except with a scaling for a difference in brightness (Star $B=75 \%$ Star $A$ ). With this assumption, reasonable fits to $\mathrm{N} \mathrm{V} \lambda 4944$, He II $\lambda 6036(\mathrm{n}=21$ to $\mathrm{n}=5)$, He II $\lambda 6170(\mathrm{n}=18$ to $\mathrm{n}=5)$, C IV $\lambda 5805$ doublet and N IV $\lambda 4058$ near elongation (phases 0.13 and 0.80 ) in 2005-2006 were obtained. In general, however, the spectra of the two stars outside of the eclipses are not additive, and there are many spectral anomalies. For example, in the 2005 data, the same N IV $\lambda 4058$ profile/flux fits the observed data at $\phi=0.0,0.37$ and 0.8. However, at phase 0.13 there is an extra emission peak, blue-shifted from that of Star A (Foellmi et al. 2008). The shift to the blue is consistent with the emission arising in Star B, but it is unclear why extra emission (red-shifted) is not seen at phase 0.8. The $\mathrm{H} \gamma$ line also exhibits extra blue-shifted emission at phase 0.13. At phase 0.8 , the profile is broader, with enhanced emission on either side of the $\phi=0.0$ profile. The system is near periastron at phase 0.13 and thus the RV separation of lines is a maximum. In addition, the interaction processes are enhanced due to the smaller orbital separation.

Among the interaction effects, a wind-wind collision is the one that is likely to dominate. Koenigsberger (2004) discussed the manner in which the changing orbital separation

7 Even if it is not an intrinsic variable its emission line spectrum will be influenced by its companion star.

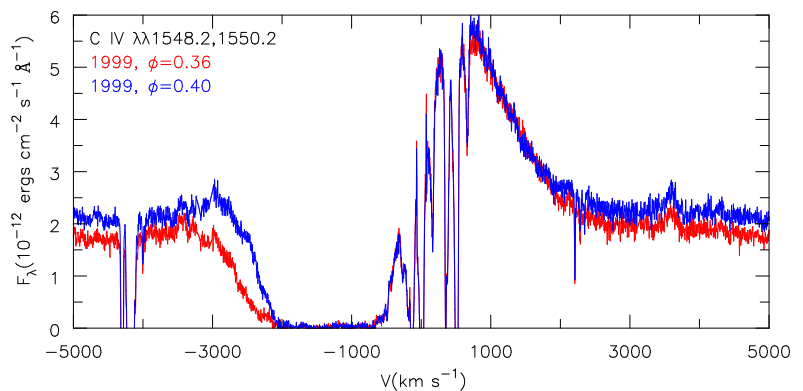

Figure 5. Comparison of C IV profiles in 1999 at phases 0.36 and 0.40. To facilitate the comparison we have subtracted off a model for Star $C$. Despite the relatively small change in phase, the maximum absorption velocity at phase 0.40 is substantially less than at phase 0.36 .

and Star A mass-loss characteristics can affect the geometry of a wind-wind shock cone. However, because of the uncertainty in the parameters of Star B, fully constraining the shock geometry is not currently possible. Furthermore, given the large mass-loss rate of each star, the shocks may not be adiabatic and will exhibit complex instabilities (Stevens et al. 1992; Nazé et al. 2018), to the extent that the shock cone geometry will be highly disrupted. Hence, our use here of the term "shock cone" should be interpreted to mean the interaction region containing the shocked winds from Star A and Star B.

These instabilities imply that spectra and photometry will exhibit random fluctuations from cycle-to-cycle. In this context, the UV spectral data taken in 1999 at phases 0.36 and 0.40 are particularly illuminating. In accord with eclipse expectations, the data at phase 0.40 are brighter than at phase 0.36 - by about $13 \%$. However, despite the relative small change in phase, the maximum absorption velocity at phase 0.40 is substantially less than at phase 0.36 (Fig. 5).

To explain this phenomenon, one could envisage that at this epoch, the wind of Star A, being substantially stronger than that of Star B, wraps around Star B. However, calculations and observations of the wind-wind collision at X-ray wavelengths (Nazé et al. 2018) both show that this is not the case, thus leaving the observed variation in P Cygni absorption components unexplained.

\subsection{Star $C$}

Although Star $C$ is also a binary, our model for this star (see Section 6), treated as though it were single, does an excellent job of removing its contaminating influence from the observed spectra. There are as yet no spectral features identified that can be associated with the companion. The presence of Star $C$ in UV spectra is best exemplified by its influence on the N V $\lambda \lambda 1238,1242$ and C IV $\lambda \lambda 1548,1552$ resonance transitions, and by the presence of photospheric transitions due, for example, to O IV $\lambda \lambda 1338,1343$ and the C III $\lambda 1176$ triplet (Georgiev et al. 2011). However, there is an indication that Star $C$ is also variable. In particular the location of the blue edge of the C IV $\lambda \lambda 1548,1552$ transition due to $S \operatorname{tar} C$ seems to be variable, and this variability cannot be eliminated by RV variations if these are fixed by the photospheric absorption line. In many of the plots we have removed Star $C$ from the observations. While there 
is still some uncertainty in the parameters and hence the spectrum of Star $C$, this procedure allows changes in fluxes and spectra of the $\mathrm{AB}$ binary to be better appreciated. In general, the "black" portions of the N V $\lambda \lambda 1238,1242$ and C IV $\lambda \lambda 1548,1552$ resonance transitions are close to zero in the corrected spectra despite the significant variations in continuum flux from epoch-to-epoch. Because of the variation in the location of the blue edge of the P Cygni profiles, small glitches in the bottom of the P Cygni profile are sometimes produced, but these do not affect the analyses. In the optical, Star $C$ reveals itself through the presence of weak photospheric O III $\lambda 5592$ and He I absorptions.

\section{SPECTRAL VARIATIONS AND FURTHER INFERENCE ABOUT Star A AND Star B}

In order to further illuminate the nature of Star A and Star $B$ we will compare spectra obtained at different epochs, and at different phases. Since the year 2000, the limited UV observations we have been able to secure have been obtained (with one exception) at orbital phases $\phi \simeq 0$, which is when Star $A$ occults Star B. Star A's wind velocity can thus be monitored since the Star $B$ wind region where P Cygni absorptions are formed is eclipsed. This strategy allows us to compare spectra of Star A obtained at different epochs, and to characterize its changing properties. Georgiev et al. (2011) analyzed spectra from epochs 1994, 2000, 2002 and 2009 using CMFGEN (Hillier \& Miller 1998), and were able to derive the mass-loss rate $\dot{M}$, total luminosity $L$, and radii and temperature at different optical depths. The basic conclusion of these analyses is that $\dot{M}$ and $L$ have been systematically declining since 1994 . At the same time, $V_{\infty}$ has been increasing.

Tables 2 and 3 list the energy contained in the most prominent lines and in the continuum bands centered at 1850, 2550, 2900, 3600 and $7360 \AA$ for the epochs 1979, 2000, 2002, 2009, 2014 and 2016. For the emission lines, this energy is the integral over the line profile above the continuum level (or below in the case of the absorptions), and typical uncertainties are $\sim 10 \%$. The line strengths of 2014 have declined to their 1979 levels. The similarity of spectra in 1979 and 2014 is shown in Fig. 2. The differences in the spectra, such as a lower continuum level and stronger He II $\lambda 1640$ (and N V $\lambda \lambda 1238,1242$, which is not shown), are likely due to the different orbital phases of the observations: $\phi=0.91$ in 1979 versus $\phi=0.00$ in 2014. A more detailed discussion of the spectral changes is presented below.

\subsection{Variations at phase $\sim 0.0$}

In Figs. $6 \& 7$ we compare data at phase zero taken in 2000 and 2014. For the UV, we have scaled the 2014 data by a factor of 1.4 to facilitate profile comparisons. The spectra are remarkably similar. While the continuum flux in the UV has decreased by $30 \%$ over 14 years, the same spectral lines are present. The noticeable changes are a significant reduction in the EW of He II $\lambda 1640$ and N IV] $\lambda 1486$, and an increase in the terminal velocity, as determined from the blueward extent of P Cygni absorption profiles. Although not easily noticeable on the plots, the fwhm of the C IV $\lambda \lambda 1548,1552$ and He II $\lambda 1640$ emission lines are somewhat larger in 2014,
Table 2. UV absolute fluxes not corrected for reddening.

\begin{tabular}{|c|c|c|c|c|c|c|c|}
\hline \multicolumn{2}{|c|}{ Line ID } & $1979^{a}$ & 2000 & 2002 & 2009 & 2014 & 2016 \\
\hline Ion & $(\AA)$ & \multicolumn{6}{|c|}{$\left|-\left(10^{-12} \mathrm{erg} \mathrm{cm}^{2} \mathrm{~s}^{-1}\right)--\right|$} \\
\hline $\mathrm{N} \mathrm{Ve}^{b}$ & 1240 & 21.0 & 29.3: & 25.6 & 21.1 & 22.4 & 11.0 \\
\hline N IV] & 1486 & $<3$ & 14.6 & 12.2 & 6.0 & 4.8 & 4.6 \\
\hline C IVa & 1550 & -18.0 & -23.7 & -21.8 & -20.5 & -20.3 & -24.2 \\
\hline C IVe & 1550 & 17.7 & 25.5 & 23.7 & 15.6 & 16.9 & 9.6 \\
\hline $\mathrm{He}$ IIa & 1640 & -3.7 & -2.3 & -6.0 & -5.0 & -3.0 & -3.1 \\
\hline He IIe & 1640 & 46.5 & 93.7 & 78.8 & 58.2 & 50.3 & 47.8 \\
\hline $\mathrm{N} \mathrm{IVa}^{c}$ & 1718 & -4.7 & -7.9 & $\ldots$ & -4.8 & -3.3 & -3.9 \\
\hline $\mathrm{N} I V \mathrm{Ie}^{c}$ & 1718 & 8.7: & 13.3 & 7.1: & 4.3 & 6.0 & 2.2 \\
\hline He II & 2385 & $\ldots$ & 2.52 & 1.91 & 0.96 & 1.0: & 0.5 \\
\hline He II & 2511 & & 3.74 & 3.22 & 1.34 & 1.76 & 1.6 \\
\hline \multirow[t]{2}{*}{ He II } & 2733 & $\ldots$ & 5.48 & 4.78 & 2.52 & 2.92 & 2.93 \\
\hline & \multicolumn{7}{|c|}{$\left|--\left(10^{-12} \mathrm{erg} \mathrm{cm}^{2} \mathrm{~s}^{-1} \AA^{-1}\right)--\right|$} \\
\hline $\mathrm{F}_{c}$ & 1850 & 1.60 & 1.68 & $\ldots$ & 1.52 & 1.38 & 1.57 \\
\hline $\mathrm{F}_{c}$ & 2550 & $\ldots$ & 0.75 & $\ldots$ & 0.66 & 0.60 & 0.69 \\
\hline $\mathrm{F}_{c}$ & 2900 & $\ldots$ & 0.55 & $\ldots$ & 0.52 & 0.47 & 0.49 \\
\hline
\end{tabular}

${ }^{a}$ The 1979 spectrum is SWP 4345 from IUE, obtained at orbital phase 0.91 .

${ }^{b}$ The N V $\lambda \lambda 1238,1242 \mathrm{EW}$ is strongly dependent on the chosen continuum level.

${ }^{c}$ The N IV $\lambda 1719$ line in 2002 is on the edge of the order.

${ }^{d}$ Uncertainties are on the order of $10 \%$. The strength of the $\mathrm{P}$ Cygni lines were measured by simultaneously fitting Gaussians to both the absorption and emission component. Strong interstellar lines do not affect the fluxes obtained by the fitting procedure. Negative fluxes indicate absorption.

Table 3. Visual fluxes not corrected for reddening.

\begin{tabular}{lcccc}
\hline \multicolumn{2}{c}{ Line ID } & 2000 & 2014 & 2016 \\
Ion & $(\AA)$ & $\left(10^{-12} \mathrm{erg} \mathrm{cm}^{2}\right.$ & $\left.\mathrm{s}^{-1}\right)$ \\
\hline HeII & 3203 & 9.54 & 5.27 & 5.42 \\
NIV & 3483 & 2.34 & 1.48 & 1.23 \\
HeII & 3923 & 0.26 & $<0.10$ & $\ldots$. \\
HeII+H & 3968 & 0.41 & 0.07 & $\ldots$. \\
HeII+HeI & 4025 & 0.53 & 0.30 & 0.06 \\
NIV & 4058 & 1.08 & 0.55 & 0.31 \\
HeII+H & 4100 & 1.94 & 0.83 & 0.47 \\
HeII & 4200 & 0.57 & 0.30 & 0.34 \\
HeII+H & 4338 & 1.38 & 0.55 & 0.49 \\
HeII & 4541 & 0.97 & 0.38 & 0.33 \\
HeII & 4686 & 14.1 & 7.76 & 8.14 \\
HeII+H & 4859 & 2.24 & 1.13 & 1.11 \\
HeII & 5411 & 1.68 & 0.98 & 0.93 \\
CIV & 5806 & 0.67 & 0.28 & 0.23 \\
HeI+HeII & 5875 & 0.68 & 0.23 & 0.27 \\
HeII & 6170 & 0.08 & $\ldots$ & 0.01 \\
HeII+H & 6560 & 3.96 & 2.06 & 1.96 \\
HeIIbl & 6683 & 0.56 & 0.30 & 0.23 \\
HeII & 6890 & 0.30 & 0.14 & 0.16 \\
NIV & 7120 & 1.15 & 0.57 & 0.45 \\
HeII & 7177 & 0.60 & 0.22 & 0.28 \\
HeII & 7592 & 0.47 & 0.25 & 0.20 \\
HeII & 8236 & 0.48 & 0.28 & 0.23 \\
\hline & & $\left(10^{-12} \mathrm{erg} \mathrm{cm}^{2} \mathrm{~s}^{-1} \AA^{-1}\right)$ \\
\hline F & 3600 & 0.31 & 0.24 & 0.26 \\
$\mathrm{~F}_{c}$ & 7360 & 0.039 & 0.026 & 0.028 \\
\hline
\end{tabular}



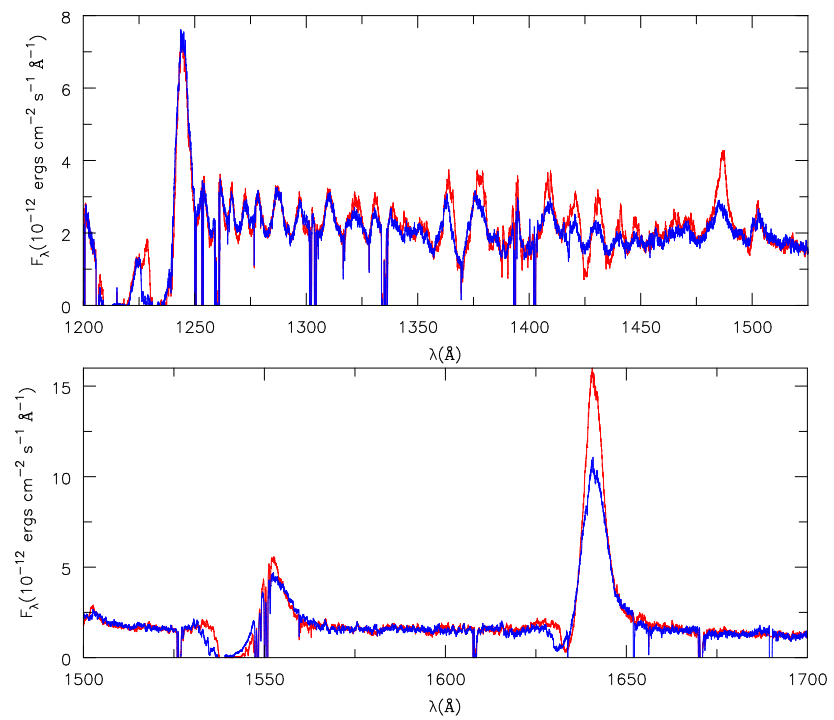

Figure 6. Comparison of the spectrum obtained in 2000 (red) with the spectrum obtained in 2014 (blue). To facilitate the comparison we have subtracted off a model for Star $C$, and scaled the 2014 spectrum by a factor of 1.4 . The most striking changes are (i) the decline in strength of N IV] $\lambda 1486$ and He II $\lambda 1640$ between 2000 and 2014 (in both absolute flux and EW) and (ii) the higher velocity absorption seen on $\mathrm{N} \mathrm{V} \lambda \lambda 1238,1242$, C IV $\lambda \lambda 1548,1552$, and He II $\lambda 1640$.

although not by as much as would be expected by the enhancement in $V_{\infty}$. Spectra taken in 2009 are very similar to those in 2014, except for a larger flux and an $V_{\infty}$ intermediate between 2000 and 2014. Thus the (apparent) increase in $V_{\infty}$ discussed by Georgiev et al. (2011) has continued.

One surprising aspect is that between 2000 and 2009 the UV continuum flux $(\lambda<1700)$ changed less than 5\% - the main change was a reduction in line strengths. The somewhat larger change in the near UV optical fluxes is probably due to the larger contribution of emission from the wind at these wavelengths, However, between 2009 and 2014 the spectrum is similar while the UV flux (in lines and the continuum) has gone down by $30 \%$, indicative of a drop in the luminosity of the system.

For the comparison at longer wavelengths, we use rectified data. As readily evident from Fig. 7 the spectra are very similar. In general the line EWs have decreased, although the variation is not the same for all lines. The EW of N IV $\lambda 3481$ and N IV $\lambda \lambda 7103-7127$ have remained relatively constant, while N IV $\lambda 4058$ and C IV $\lambda \lambda 5801,5812$ have decreased. To further aid our understanding of the spectral changes, we plot the ratio of the 2000 data relative to the 2014 data in Fig. 8. As apparent from this figure, the energy distribution in 2014 is much bluer than in 2000. This change is consistent with a decline in wind density between 2000 and 2014.

We attribute the bump in Fig. 8, centered at $\sim 2030 \AA$, to extra emission in the He II n-3 series as we approach the series limit at $2050 \AA$, and a different slope in the $\mathrm{He} \mathrm{II}(\mathrm{n}=3)$ continuum. The bump is close to the interstellar $\lambda 2150$ bump, but is blue-shifted. In principle, we could get a bump due to extinction if Star $C$ were not associated with Star A and Star B, since the effective extinction would change with the relative brightness of Star C to Star A and Star B. In the fitting we have assumed a single extinction for all stars.

\section{$\mathbf{5 . 2}$ Variations at phase $\sim \mathbf{0 . 3 6}$}

At phase $0.36 \operatorname{Star} A$ is eclipsed. While we have fewer UV observations at this phase, they are important for understanding the system. Since we know that the strength of Star A's wind has been declining since 1999, we might expect to see substantial differences between the old data, and the data (at phase 0.36) obtained in 2016. Because the eclipses are not total, it is also important to compare data taken at phase $\sim 0.36$ with that taken near phase $\sim 0.0$.

A comparison of UV spectra at phase 0.37 in 1999 and at phase 0.0 in 2000 (Fig. 9) shows spectra (and fluxes) that are remarkably similar - the primary differences are that the absorption on N V $\lambda \lambda 1238,1242$, C IV $\lambda \lambda 1548,1552$, and He II $\lambda 1640$ extends to higher velocities in the 1999 data, and the strength of the Fe V/Fe VI forest between 1200 and $1500 \AA$. The weakening of the Fe forest is consistent with that seen in the simple "occulting-disc model" discussed in Sect. 4.2. The similarity suggests that most of the emission spectrum in 1999 at phase 0.36 is associated with Star A. This is confirmed when we compare 1999 data (phase 0.36) with 2016 data (phase 0.35) (Fig. 10).

nivinter is much stronger in the 1999 data - its weakness in the 2016 data is consistent with the change in the spectrum of Star A. In 1999 the continuum level is about 10 to $20 \%$ larger than in 2016 (with Star $C$ subtracted off) and the emission lines (e.g., He II $\lambda 1640$ and C IV $\lambda \lambda 1548,1552$ ) exhibit both a larger flux and a larger EW.

The data taken in 2016 show several spectral anomalies (Figs. $11 \&$ 12). First, many of the profiles (including the emission components of the resonance transitions) are asymmetric. This asymmetry is readily apparent, even in our low resolution optical data obtained with HST. Second, the emission profiles indicate substantially lower velocities than implied by the extent of the P Cygni absorption profiles associated with C IV $\lambda \lambda 1548,1552$ and N V $\lambda \lambda 1238,1242$. Third, the absorption profiles associated with C IV $\lambda \lambda 1548,1552$ and N V $\lambda \lambda 1238,1242$ show an anomalous structure. In particular, the emission does not merge smoothly with the absorption. In the C IV $\lambda \lambda 1548,1552$ profile enhanced absorption commences abruptly near $-1000 \mathrm{~km} \mathrm{~s}^{-1}$, while in the $\mathrm{N} \mathrm{V} \lambda \lambda 1238,1242$ profile the absorption commences abruptly near $-500 \mathrm{~km} \mathrm{~s}^{-1}$ (Fig. 11). The difference between the absorption onset velocities is due to the doublet nature of these two resonance transitions. The doublet separation for C IV is $\sim 500 \mathrm{~km} \mathrm{~s}^{-1}$, while for N V it is $\sim 960 \mathrm{~km} \mathrm{~s}^{-1}$. A further surprising aspect is the similarity of the data obtained in 2014 at phase 0 (Star B eclipsed) and in 2016 at phase 0.35 (Star A eclipsed), as illustrated in Fig 12. However, there is one striking difference: $\mathrm{N} \mathrm{V} \lambda \lambda 1238,1242$ is much weaker in the 2016 data set. The same similarity was noted in the 1999 data, but in 2014/2016 the radius of Star $A$ is smaller than in 1999, and should be much more similar to that of Star B.

For He II $\lambda 1640$ the asymmetry appears to be indicative of missing emission rather than enhanced blue-shifted emission. For C IV $\lambda \lambda 1548,1552$ the situation is less clear there is less red-shifted emission but also more blue-shifted 


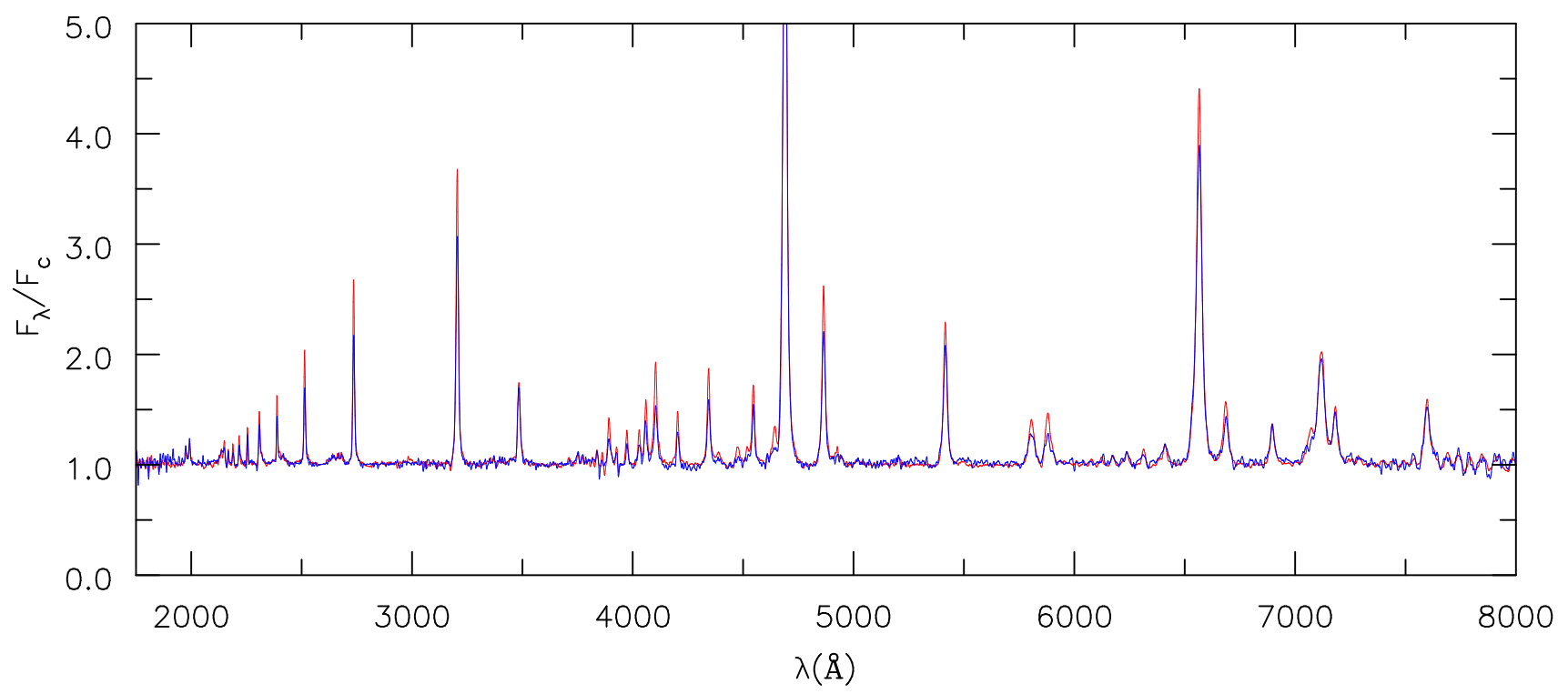

Figure 7. Comparison of spectra obtained in 2000 (red) with that obtained in 2014 (blue). Both spectra were obtained at phase 0.0 when Star B is eclipsed. While there is a significant decline in line EWs between 2000 and 2014 the spectra are quite similar.

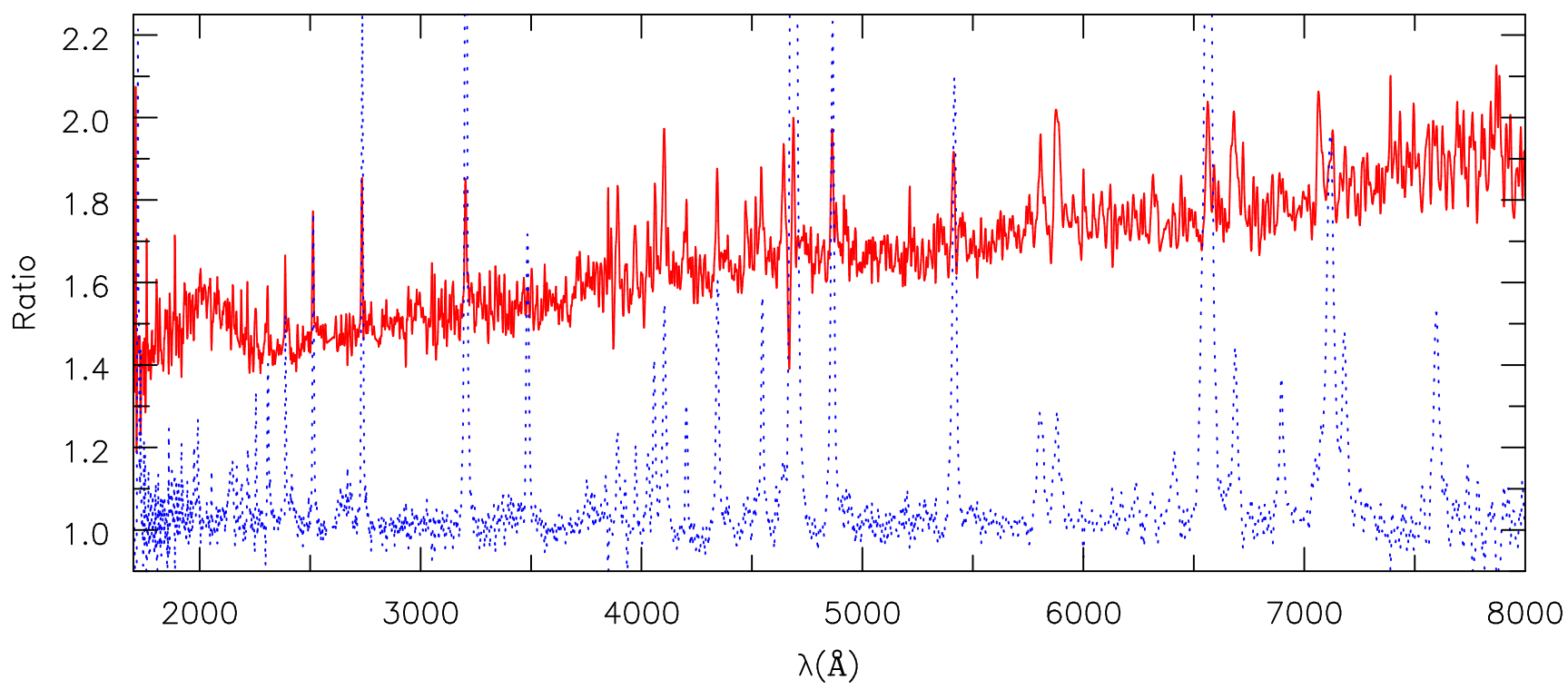

Figure 8. Flux ratio of the STIS spectra obtained in 2000 and 2014 illustrating that the 2000 spectrum was significantly redder than in 2014, and that the apparent emission "bump" at $2000 \AA$ was also significantly stronger in 2000 than in 2014. The dotted tracing is the normalized spectrum of 2014. Comparison of this spectrum with the ratio spectrum also shows that the EWs of most emission lines were larger in 2000. Particularly striking is the much stronger C IV $\lambda \lambda$ 5801, 5812 and He I $\lambda 5876$ emission in 2000. These features are amongst the strongest in the ratio spectrum. To compute the ratio spectrum we subtracted the contribution of Star $C$, and smoothed over several interstellar features in the UV.

emission. Further, N V $\lambda \lambda 1238,1242$ does not exhibit the blueshifted emission profile.

We examine the 2016 data further in Section 7.2. In that section we also discuss the implications of the 2016 data for understanding the HD 5980 system.

\section{CMFGEN FIT TO THE 2014 DATA}

We proceed as in Georgiev et al. (2011) by assuming that Star $B$ is completely hidden from view at the $\phi=0$ eclipse. The adopted spectrum for $\operatorname{Star} C$ (which is always in view) is taken into account and assumed to be that of a O67 supergiant, as implied by the photospheric absorptions (Koenigsberger et al. 2002). The parameters adopted for Star $C$ are the same as those found by Georgiev et al. (2011). The contribution of this star to the emission lines is minimal except for C IV $\lambda \lambda 1548,1552$. The absence of a significant contribution to N V $\lambda \lambda 1238,1242$ (the supergiant actually shows very weak and unblended $\mathrm{P}$ Cygni profiles in $\mathrm{N} \mathrm{V} \lambda \lambda 1238,1242$ ) and the contribution to the absorption component C IV $\lambda \lambda 1548,1552$ can easily be discerned (Figures 1, 2 \& 3 in Georgiev et al. (2011), Fig. 14). 


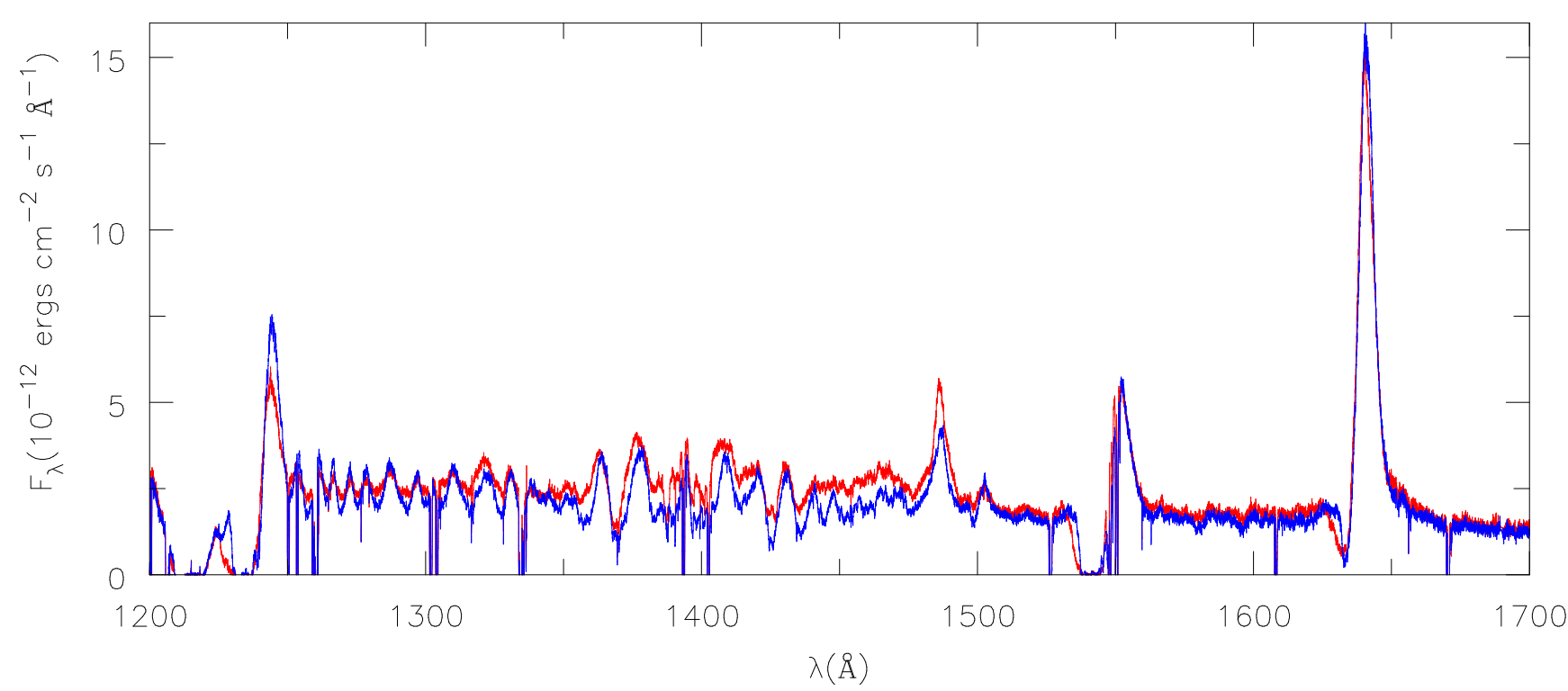

Figure 9. Comparison of data at phase 0.36 (red) obtained in 1999 (when Star $A$ is eclipsed) and at phase 0.0 in 2000 (blue) when Star $B$ is eclipsed. The spectra are remarkably similar - the primary differences are that the absorption extends to higher velocities in the 1999 data and the strength of the Fe VI/Fe V forest between 1200 and $1500 \AA$. No scaling of the data has been performed, and to facilitate the comparison we have subtracted off a model for Star C.

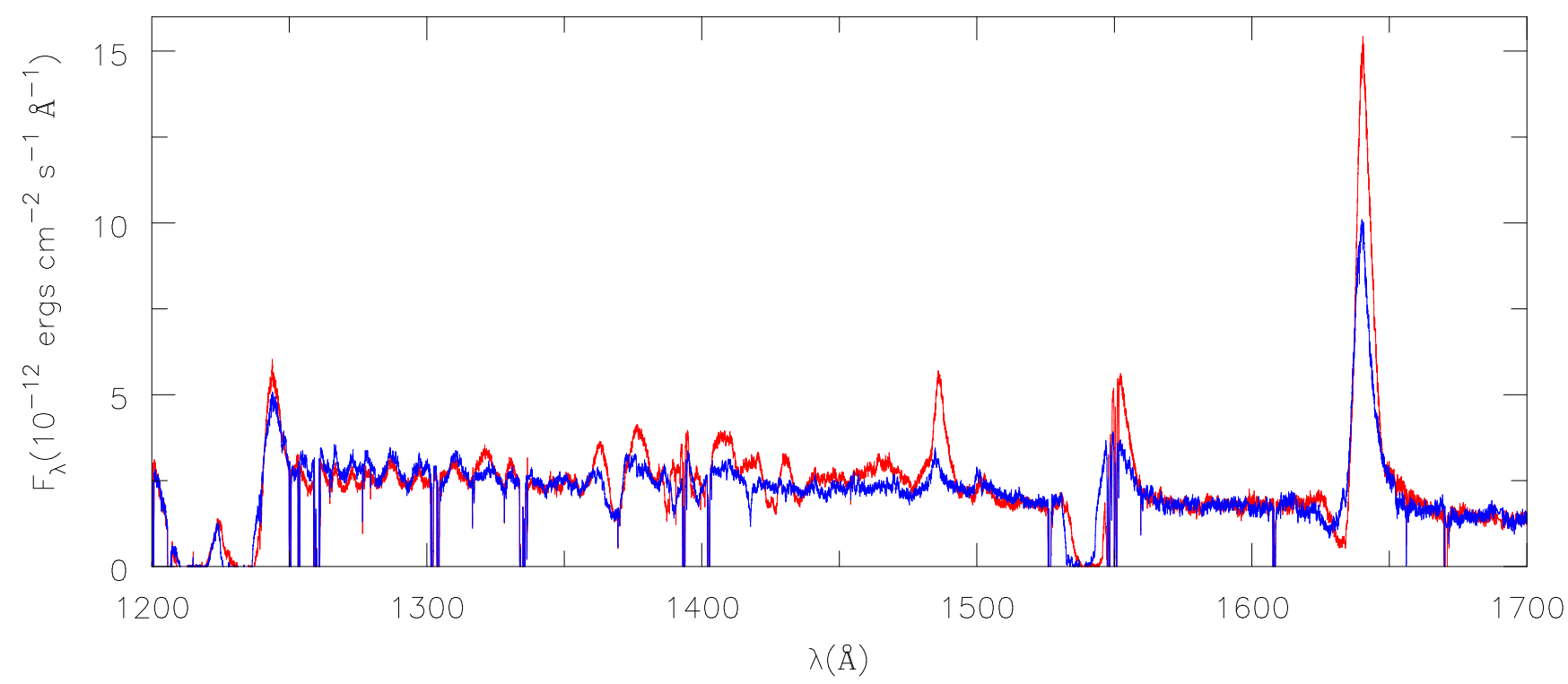

Figure 10. Comparison of data obtained at phase 0.36 in 1999 (red) and phase 0.35 in 2016 (blue) when Star A is eclipsed. To facilitate the comparison we have subtracted off a model for Star $C$, and we have scaled the 2016 data by a factor of 1.2 , although near $\lambda 1200$ a factor of 1.1 would give better agreement between the two continua.

For the modeling we adopt a distance of $64 \mathrm{kpc}$ (the same as adopted by Georgiev et al. (2011)) - the current best estimate for the distance to the SMC is approximately $62 \mathrm{kpc}$ (Hilditch et al. 2005; Scowcroft et al. 2016), although due to the tilt and depth of the SMC there will be some variation in the distance of individual stars (Scowcroft et al. 2016). For the reddening we adopt E(B$\mathrm{V})=0.05$ for the galaxy contribution and 0.025 for the SMC. The total reddening is similar to the value of 0.07 found by Koenigsberger et al. (1998b) and 0.05 derived by Schmutz \& Vacca (1991). To redden the model spectra we use the extinction law of Cardelli et al. (1989) with $\mathrm{R}=3.1$ for the Galaxy and for the SMC. Slight changes in the reddening parameters will alter the fit quality. In practice it is difficult to get an accurate fit (i.e. to a few percent) over both the optical and the UV. This is likely due to a combination of several factors - uncertainties in the amount of reddening and the reddening laws, uncertainties in the contribution of Star $C$ (and Star B), errors in the parameters derived for Star A, and the assumption of spherical symmetry.

The results derived from the model fit to the 2014 data are listed in Column 7 of Table 4, and compared with the 

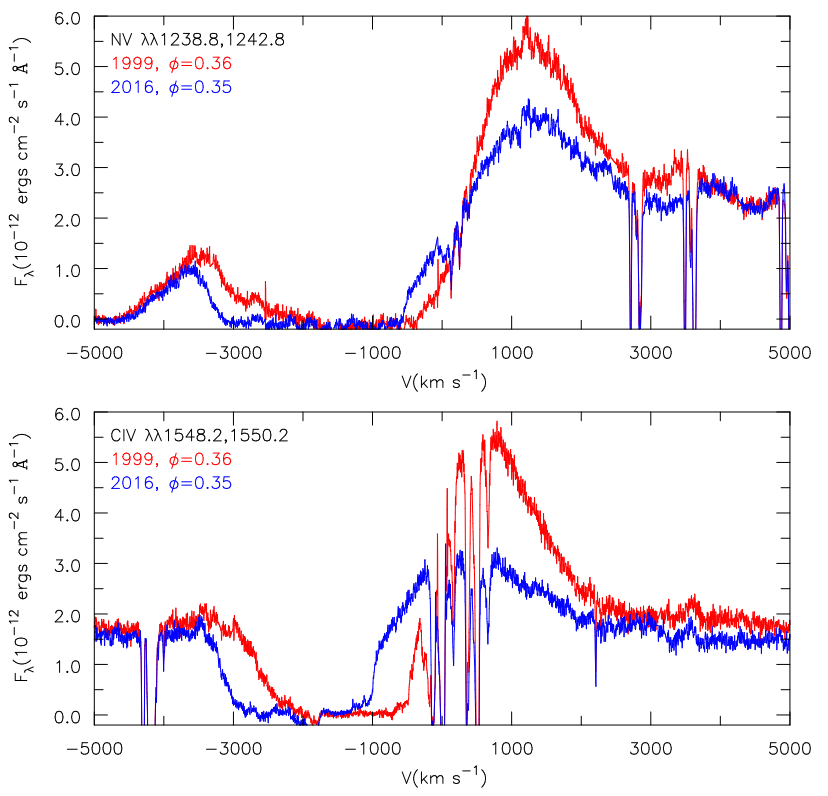

Figure 11. Comparison of N V and C IV profiles in 1999 and 2016 when Star $A$ is eclipsed. To facilitate the comparison we have subtracted off a model for Star $C$. Due to changes in the extent of the P Cygni absorption in Star $C$, and noise, the absorption profiles dip slightly below zero. Both the N V and C IV profiles exhibit significantly higher absorption velocities in 2016 . In addition, the 2016 profiles exhibit unusual P Cygni profiles: there is a sudden onset of absorption as we move towards the blue from line center. In $\mathrm{N} \mathrm{V}$ the onset occurs at $\sim 500 \mathrm{~km} \mathrm{~s}^{-1}$ while in C IV it occurs at $\sim 1000 \mathrm{~km} \mathrm{~s}^{-1}$.

results of Georgiev et al. (2011) for the other epochs. Also listed in the table are the results for both Star A and Star B from Shenar et al. (2016). Koenigsberger et al. (2014) found that a simple scaling of the 2009 model for Star $A$ by 0.75 (i.e., the V-band flux ratio) provided a reasonable fit to the combined Star A plus Star B spectra outside eclipse. Due to large uncertainties in determining the intrinsic spectrum of Star $B$ we do not provide new estimates for the parameters of Star B. Note that there is a small difference in the parameters given in this paper and Nazé et al. (2018). The results in the Nazé et al. (2018) paper are for a terminal velocity of $3000 \mathrm{~km} \mathrm{~s}^{-1}$. This velocity matches the highest velocities seen in the C IV $\lambda \lambda 1548,1552$ and N V $\lambda \lambda 1238,1242$ profiles, although the model absorption of He II $\lambda 1640$ is too broad. In addition, the optical emission profiles are also somewhat too broad. In this table, $R_{10}$ and $R_{2 / 3}$ correspond to the radii at which the continuum optical depth $\tau=10$ and $\tau=2 / 3$, respectively. Similarly, $T_{*}$ and $T_{\text {eff }}$ are the temperatures at $R_{10}$ and $R_{2 / 3}$, respectively.

The 2014 entire spectral energy distribution obtained with HST/STIS is plotted in Fig. 13. The very good agreement is confirmed by inspection of the amplified segments shown in Figs. $14 \& 15$.

The value of $R_{2 / 3}=24.2 R_{\odot}$ in 2014 is close to the value of $23.6 R_{\odot}$ derived from the continuum eclipse light curve of the system observed in 1979 by Perrier et al. (2009) if we adopt $a=150 R_{\odot}$ for the orbital major semi-axis from Koenigsberger et al. (2014). This is consistent with the conclusion drawn above that HD 5980 seems to have reverted to its quiescent state similar to that in 1979. Furthermore,
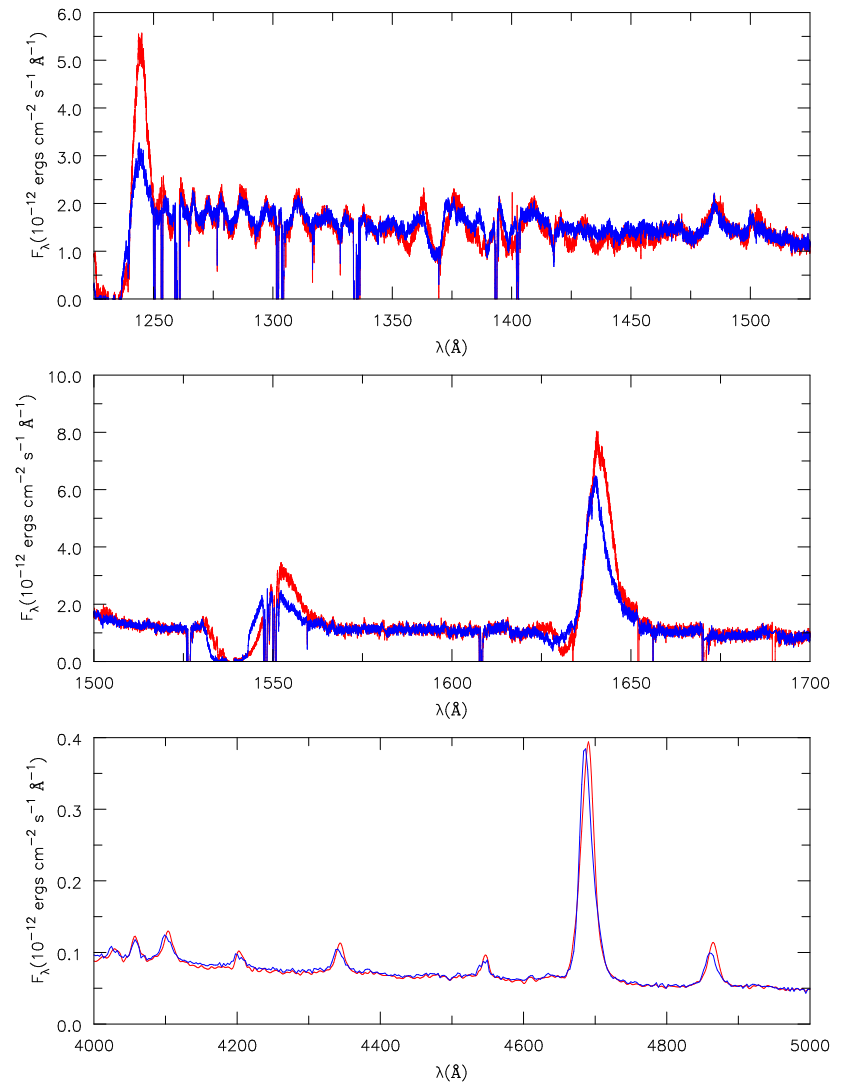

Figure 12. Comparison between the HST spectrum obtained in 2014 (phase 0.0, red) and 2016 (phase 0.35 , blue). For display purposes the 2016 UV spectrum has been multiplied by 0.75 , and the 2016 optical spectrum by 0.89 .
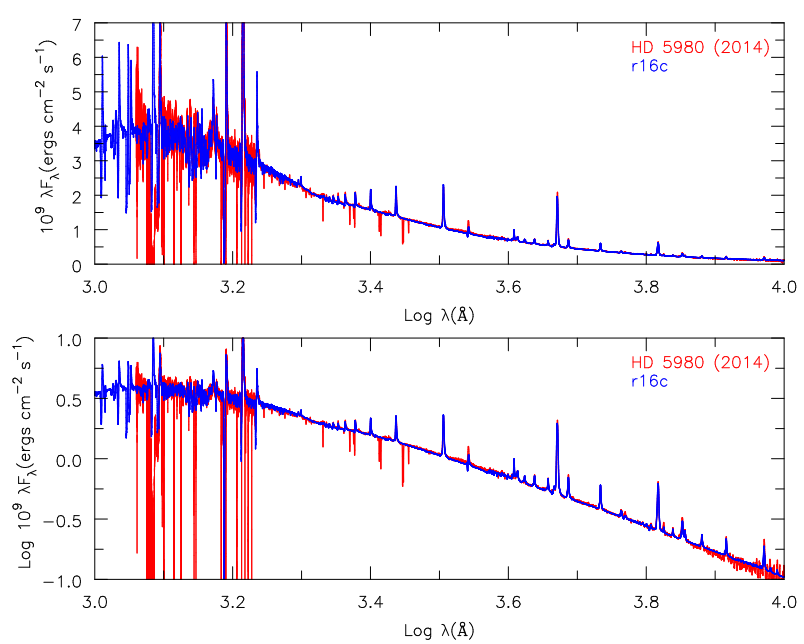

Figure 13. The spectral energy distribution of HD 5980 at orbital phase 0 obtained with HST/STIS in 2014 (red) compared with the CMFGEN model that provides the best fit. To better illustrate the distribution we use both linear (top) and logarithmic ordinate scales. 
Table 4. Model fits.

\begin{tabular}{|c|c|c|c|c|c|c|c|c|}
\hline Parameter & $09 / 1994^{a}$ & $12 / 1994^{b}$ & $2000^{b}$ & $2002^{b}$ & $2009^{b}$ & $2009^{d}$ & $2014^{c}$ & B- $2009^{d}$ \\
\hline$J D-240000.0$ & $\cdots$ & 49716.6 & 51655.1 & 52386.6 & 55083.9 & $\ldots$ & 56740.9 & $\ldots$ \\
\hline$V[m a g]$ (system) & $\ldots$ & 11.12 & 11.3 & 11.6 & $\ldots$ & $\ldots$ & $\ldots$ & $\ldots$ \\
\hline$R_{10}\left[R_{\odot}\right]$ & $\ldots$ & 28 & 20.4 & 21 & 19.3 & $24_{-7}^{+10}$ & 18.9 & $22_{10}^{+15}$ \\
\hline $\mathrm{R}_{2 / 3}\left[R_{\odot}\right]$ & 280 & 124 & 34 & 32 & 28 & $26 \underbrace{+10}_{-7}$ & $24.2^{e}$ & $23 \begin{array}{l}+15 \\
10\end{array}$ \\
\hline$T_{e f f}[k K]$ & $\ldots$ & 23 & 37.3 & 40 & 43 & $\ldots$ & 43 & $\ldots$ \\
\hline$T_{*}[k K]$ & 23 & 47 & 48 & 50 & 47 & $\ldots$ & 48 & $\ldots$ \\
\hline$\dot{M} / \sqrt{f}\left[10^{-5} M_{\odot} / y r\right]^{f}$ & 80 & 111 & 35 & 25 & 23 & $20 \pm 5$ & 14 & $10_{-10}^{+10}$ \\
\hline $\mathrm{f}$ & 0.1 & 0.025 & 0.025 & & 0.025 & 0.025 & 0.1 & 0.1 \\
\hline $\log \left(L / L_{\odot}\right)$ & 7.05 & 6.57 & 6.30 & 6.39 & 6.39 & $6.31 \pm 0.1$ & 6.23 & $6.25 \pm 0.15$ \\
\hline $\mathrm{V}_{\infty}\left[\mathrm{km} \mathrm{s}^{-1}\right]$ & 500 & 750 & 2000 & 2200 & 2440 & $2200 \pm 200$ & 2100 & $1650 \pm 100$ \\
\hline$\Gamma$ & 0.75 & 0.75 & 0.53 & 0.53 & 0.53 & $\ldots$ & $(0.33)$ & $\ldots$ \\
\hline
\end{tabular}

a Drissen et al. (2001)

b Georgiev et al. (2011)

c This paper

d Shenar et al. (2016)

e Consistent with $R_{A}=24$ from value of $R_{A} / a$ from Perrier et al. (2009) and $a$ from orbital solution of Koenigsberger et al. (2014).

f $f$ is the volume filling factor and $\Gamma$ is $L / L_{\mathrm{Edd}}$ where $L_{\mathrm{Edd}}$ is the Eddington luminosity.

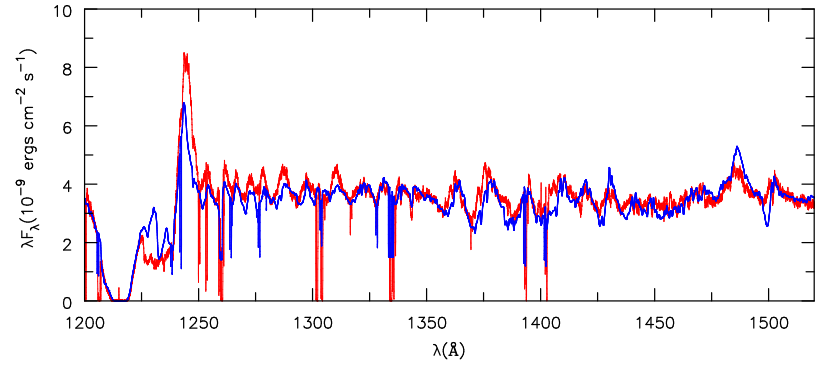

Figure 14. Illustration of the fit (blue) in the UV. Both the N V $\lambda \lambda 1238,1242$ doublet and the Fe VI forest (1250 to $1320 \AA$ ) are somewhat weaker than observed. The plateau in the observed spectrum (red) is due to a lack of $\mathrm{N} \mathrm{V}$ in the O supergiant whose theoretical spectrum is included in the fit.

it now has the smallest mass-loss rate and luminosity since 1994. These parameters have never been determined for the quiescent state.

The errors in the parameter determinations are difficult to determine since they are correlated, and since the fit quality is subjective. Even if we were to use a quantitative measure of the fit quality the errors would be subjective how do we interpret/quantify the errors when some feature cannot be fitted for any reasonable model? Below we first consider errors arising from the CMFGEN fitting. Systematic errors, arising from assumptions about the system, will be addressed later.

The fit to the 2014 spectrum shown in Figs. 13-16 corresponds to $\mathrm{V}_{\infty}=2100 \mathrm{~km} \mathrm{~s}^{-1}$. However, as can clearly be seen in Fig. 16, this value severely underestimates the extent of the P Cygni absorption components of C IV $\lambda \lambda 1548,1552$. It also underestimates $\mathrm{N} \mathrm{V} \lambda \lambda 1238,1242$ and, to a lesser extent, He II $\lambda 1640$. As the absorption component traces material along only our line of sight it may not provide the best measure of the "average wind" terminal velocity. Unfortunately it is difficult to estimate $V_{\infty}$ from the emission width with our slow velocity law - values ranging from 2000 to $2500 \mathrm{~km} \mathrm{~s}^{-1}$ are equally compatible with the emission width.

To fit the width of He II $\lambda 4686$ we needed a slow (.i.e.,
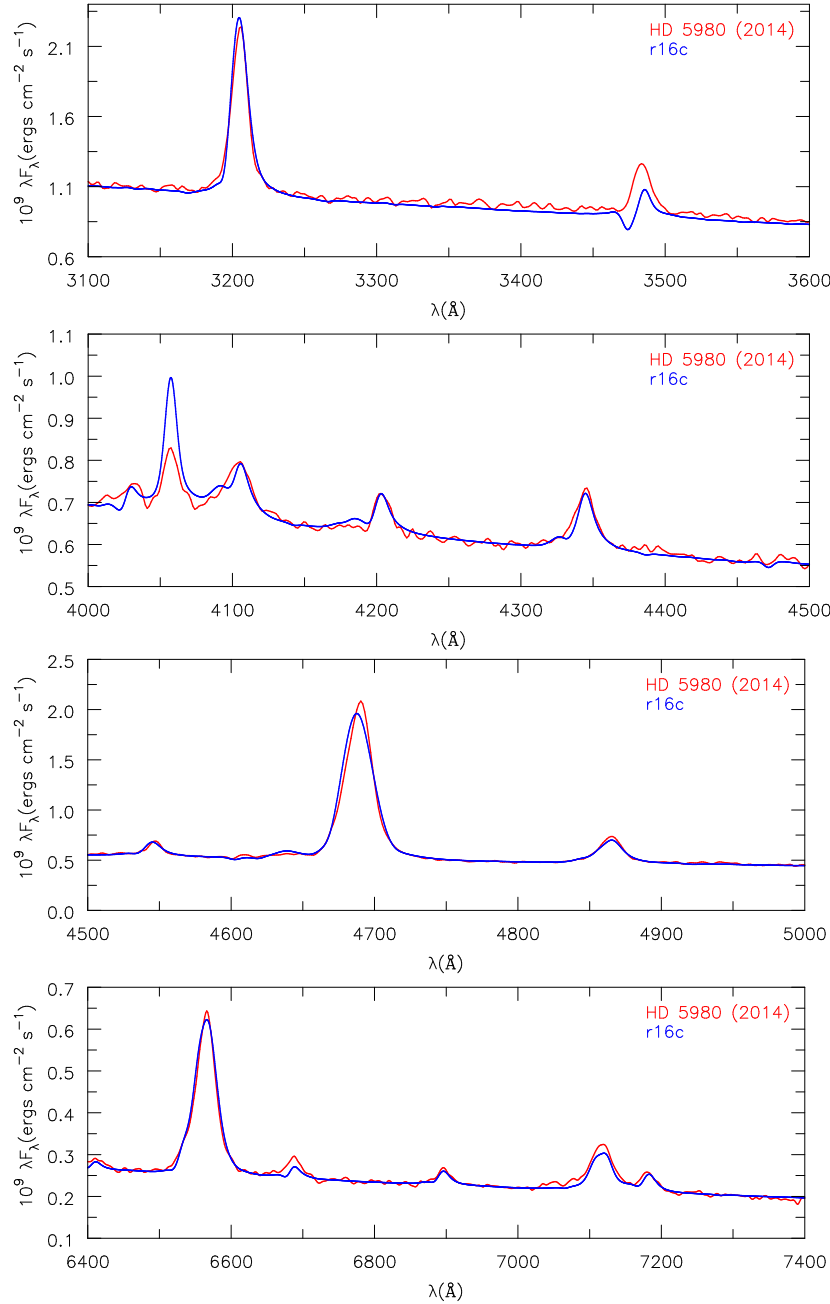

Figure 15. Amplified portions of the data shown in Fig. 13. The largest discrepancies are with the N IV lines - N IV $\lambda 4058$ is too strong in the model while N IV $\lambda \lambda 3479$ - 3485 has a P Cygni absorption component which is not seen in the low-resolution data. 

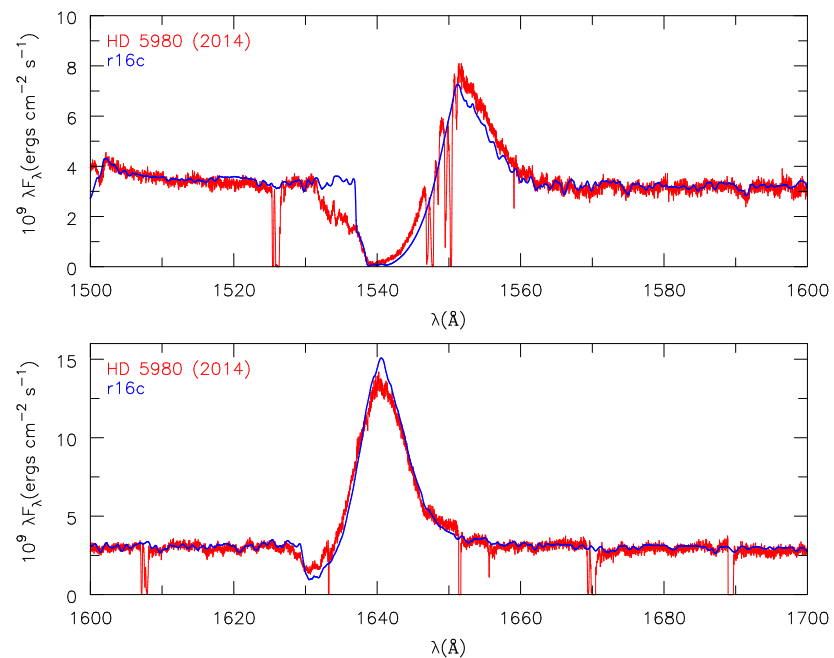

Figure 16. The line profiles of C IV $\lambda \lambda 1548,1552$ and He II $\lambda 1640$ observed in 2014 (red) compared with the same CMFGEN model shown in the previous figures. At this epoch the terminal velocity of the O supergiant is similar to what we used for Star $A$.

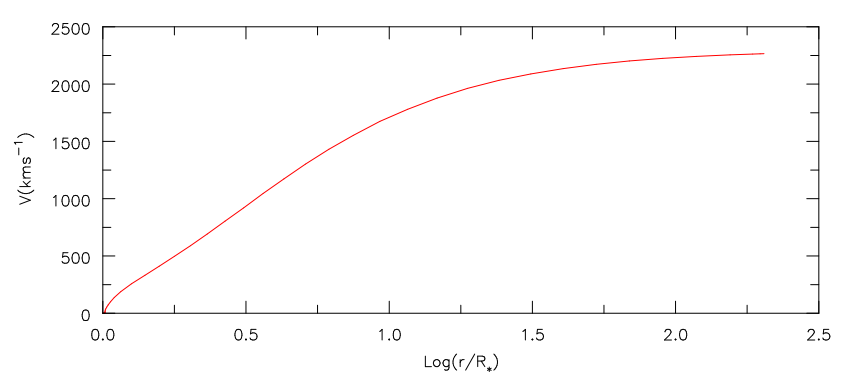

Figure 17. The velocity law used in the modeling. To avoid very low velocity gradients near the sonic point the velocity law has $\beta=1$ near the sonic point but transitions to $\beta=3$ at larger radii.

$\beta=2$ or 3 ) velocity law $-\beta=1$ gives He II lines that are too broad. Our unusual velocity law (Fig. 17) also seems to give better consistency between the emission lines and the optical continuum. Is there some effect arising from the binary interaction, such as radiative braking or mutual interaction, that necessitates a slow velocity law? Non-standard velocity laws were seen in early hydrodynamic models for WR stars (e.g Schmutz 1997). More recent modeling also gives slower acceleration due to ionization changes in the stellar wind (Gräfener \& Hamann 2005).

A major issue with our modeling is that there is an inconsistency between the velocity law and the momentum deposited in the wind - too little momentum is deposited in our model. In one sense this is not surprising. For our model the ratio of wind momentum to radiation momentum $\left(\dot{M} V_{\infty} /(L / c)\right)$ is 3.2 . This is substantially larger than determined for other WR stars in the SMC, all of which have values less than unity (Hainich et al. 2015). Changing the velocity law so that it more closely resembles a classic $\beta=1$ velocity law does not help since we would need either to enhance $\dot{M}$ or reduce the volume filling factor in order to retain a fit to emission line strengths.

From the fits we deduce that $f<0.2$, with an indeterminate lower limit. $\dot{M} / \sqrt{f}$ is derived from fitting the emis- sion strengths and is known to better than $30 \%$ for a fixed velocity law (i.e., $\left.\left(10<\dot{M} / 1.0 \times 10^{-5}\right) / \sqrt{f}<18\right)$. However, if you increase $\beta$ in the line forming region the derived $\dot{M} / \sqrt{f}$ will decrease. In principle $\dot{M} / \sqrt{(f)}$ also scales with $V_{\infty}$, but $V(r)$ in the $\mathrm{He} / \mathrm{H}$ emitting region is constrained by the profile fits. Conservatively, the fitting indicates that $40000 \mathrm{~K}<$ Teff $<50000 \mathrm{~K}$. The lower limit is set primarily by the strength of the He I lines, which become too strong at lower temperatures. The upper limit comes from a number of factors - the quality of fit near $52000 \mathrm{~K}$ is noticeably worse, especially in the UV. $T_{*}$ (the effective temperature at $\tau=10$ ) is poorly constrained, since it lies below the optically thick wind - its value will depend on the density structure below the sonic point which in turn is sensitive to the closeness of the star to the Eddington limit. The luminosity is constrained with 0.15 dex.

In addition to fitting errors, it is necessary to examine systematic errors. All evidence suggests that the system is returning to its pre-outburst state, and hence is in a state approaching that modeled by Perrier et al. (2009). With the smaller radius for Star A we expect Star B to contribute to the primary eclipse spectra. We can estimate the influence of Star B on our results as follows:

1. Assume Star B has an identical spectrum to Star A, and contributes $20 \%$ to the light/spectra at primary eclipse. With this assumption the effects on the results are rather small - the effective temperature would remain the same, the radius would be reduced by a factor of 0.9 , and the mass loss by a factor of 0.85 . The latter is a result of the use of a scaling relation - similar spectra are obtained when $\dot{M} / R_{*}{ }^{1.5}$ is preserved.

2. Assume Star B contributes $20 \%$ to the continuum at primary eclipse, but a larger fraction to the emission lines (again with a similar spectrum to Star A). If we assume that $40 \%$ of the line flux is due to Star B, then we need to reduce $\dot{M}$ by a factor of $\sim 0.67$ (assuming $\mathrm{R}$ is reduced by a factor of 0.9 to give a $20 \%$ reduction in the continuum flux).

\section{THE UV P CYGNI PROFILES}

In this section we discuss the extended absorption troughs on UV transitions which appear to indicate wind terminal velocities that are inconsistent with the optical and UV emission line widths. We also discuss the unusual P Cygni profiles observed in 2016 when Star A was eclipsed by Star B.

\subsection{Extended P Cygni absorption}

The CMFGEN models provide an excellent agreement to the overall spectral energy distribution and a majority of the emission line strengths. However, there is a discrepancy in the fit to the $\mathrm{P}$ Cygni absorption components of the UV resonance lines when we constrain the velocity law by the emission line widths. Specifically, we observe a plateau in the $\mathrm{N} \mathrm{V}$ and C IV absorption components that extends out to $-3000 \mathrm{~km} \mathrm{~s}^{-1}$, whereas $\mathrm{V}_{\infty}^{A} \leq 2500 \mathrm{~km} \mathrm{~s}^{-1}$ is derived from the CMFGEN model fit.

Anomalous P Cygni absorption components have been observed previously in HD 5980's spectra. The first documented example can be found in IUE spectra obtained shortly after the 1994 eruption in the Si IV $\lambda \lambda 1394,1403$ 
P Cygni absorption component. While lower ionization atomic species such as Al III and Si II display a maximum extent $<800 \mathrm{~km} \mathrm{~s}^{-1}$, the Si IV doublet has a maximum extent of $\sim 1700 \mathrm{~km} \mathrm{~s}^{-1}$ (González \& Koenigsberger 2014). This phenomenon was interpreted in terms of a "fossil" wind which left the system prior to the rapid decline in wind speed associated with the eruption, an interpretation that is supported by the observations which indicate that the wind speed of Star $A$ just prior to the eruption was $\sim 1700 \mathrm{~km} \mathrm{~s}^{-1}$ and that the column density remains high enough for detection a long time after the material has left the system.

The second documented example is found in the 1999 HST/STIS FUV spectrum obtained at $\phi=0.36$ in which the wings of the $\mathrm{P}$ Cygni absorptions indicated wind speeds of $\sim 3000 \mathrm{~km} \mathrm{~s}^{-1}$. This is $\sim 800 \mathrm{~km} \mathrm{~s}^{-1}$ faster than observed at the other orbital phases when Star B is unocculted by Star $A$. The fact that Star B was in front of Star A at this time led to the interpretation that the faster wind originated in Star B, but was impossible to detect at other orbital phases due to the constraining effect of the wind-wind "shock cone" produced through the interaction with Star A's slower, but more powerful, wind. However, adopting an interpretation in terms of a "fossil" wind, it is possible that the fast wind arises in Star $A$ at some orbital phase $<0.36$.

The evolution over time of the C IV $\lambda \lambda 1548,1552$ P Cygni absorption component is shown in Fig. 18 around the eclipse phases. The progression towards a more extended and shallow absorption between 1991 and 2014 is clearly visible, as is the similarity between the 2014 and the 1979 components.

The seven IUE spectra that were observed in 1979-1981 display similarly extended P Cygni absorptions. These were obtained at different orbital phases, including 0.91 (Star A in the foreground) and 0.48 (Star B in the foreground). Only two UV spectra of HD 5980 are available for the 2014-2016 epoch. Thus, we can only speculate at this time on whether the extended absorption is present also in the current epoch at all orbital phases, and on the origin of the fast wind component and its implications. It is also interesting to note that the discrepancy was already present in 2009, but at that time we favored fitting C IV (leading to $\mathrm{V}_{\infty}^{A}=-2440 \mathrm{~km} \mathrm{~s}^{-1}$ ), and the anomalous absorption component appeared in He II $\lambda 1640$ (see Fig. 11 in Georgiev et al. (2011)). The anomaly was not evident in the fit to the 2002 STIS spectrum $\left(\mathrm{V}_{\infty}^{A}=-2200 \mathrm{~km} \mathrm{~s}^{-1}\right)$, although an anomaly was noted by Koenigsberger et al. (2006) in the FUSE line profiles of the same year. They concluded that $\mathrm{V}_{\infty}^{A} \sim 1750 \mathrm{~km} \mathrm{~s}^{-1}$, but noted the presence of additional absorption extending out to $-2200 \mathrm{~km} \mathrm{~s}^{-1}$. Hence, whatever is causing the anomaly, it is an effect that is growing in importance as Star A's massloss rate declines. The change could be related to the optical depth of Star A's wind, or the geometry of the wind-wind collision "shock cone", since both are influenced by a smaller $\dot{M}_{A}$.

There are several possible explanations for the anomalous absorption:

(i) In "single" stars extended absorption is often seen on UV P Cygni profiles and this is associated with instabilities associated with line driving. $V_{\infty}$ is usually associated with $V_{\text {black, }}$ which is the maximum blueward extent of the "flat" minimum of the saturated absorption trough. In Fig. 18 we
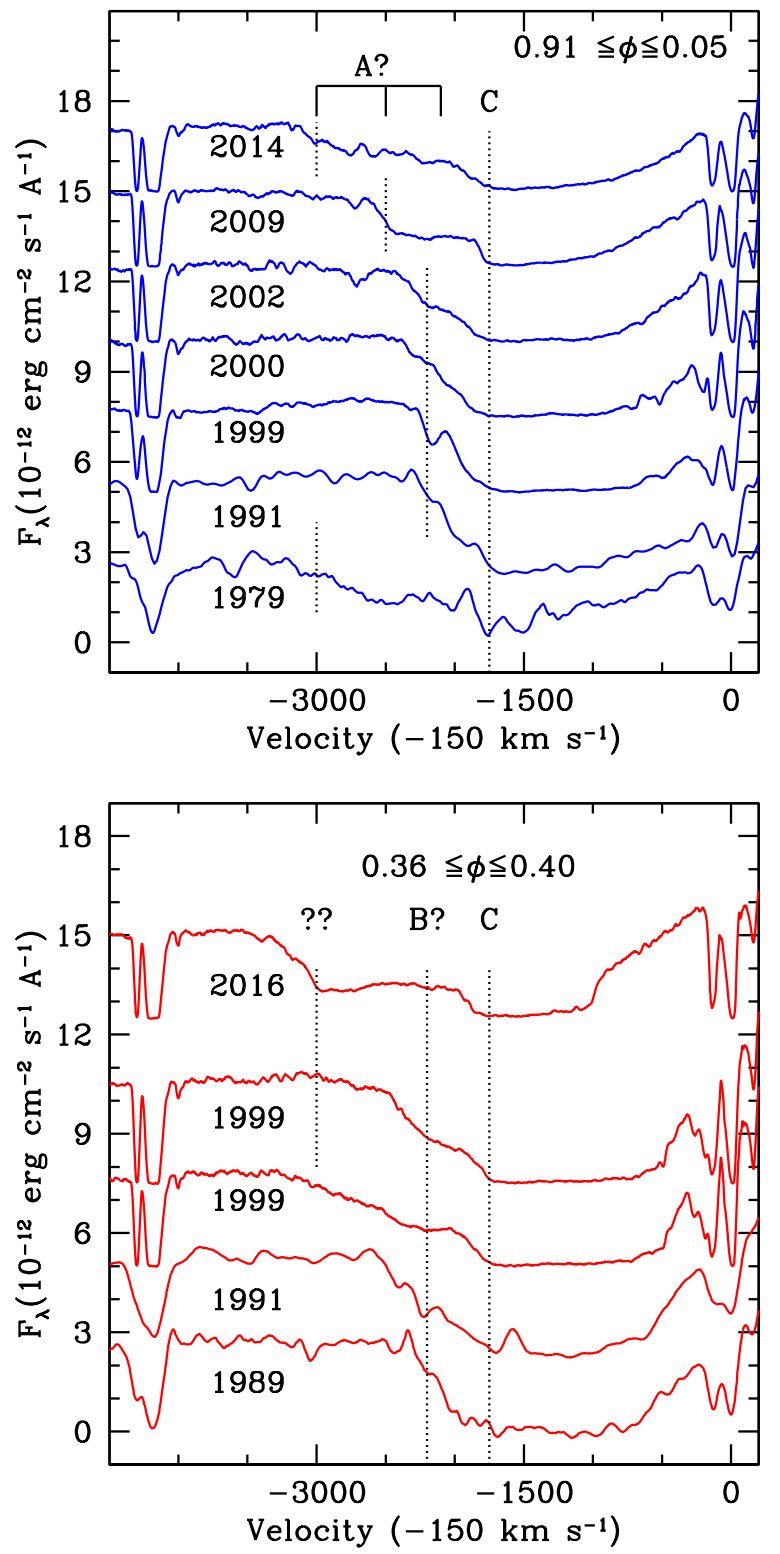

Figure 18. P Cygni absorptions of C IV $\lambda \lambda 1548,1552$ over time. Top: Orbital phases around the time when Star $A$ is in front of Star B; Bottom: Orbital phases when Star $B$ is in front. The epoch of observation is noted. The vertical line that is labelled "C" indicates the extent of the saturated absorption component of Star C. The vertical lines labelled "A" and "B" indicate the possible (and hence the ?) location of the absorption edge from Star A and Star B, respectively. The origin of the highest velocity absorption seen when $\operatorname{Star} A$ is eclipsed is uncertain, hence the ??. Each spectrum is shifted horizontally to correct for the orbital motion of Star A, and shifted vertically for clarity in the figure. The vertical shift is $n \times 2.5 \times 10^{-12} \mathrm{erg} \mathrm{cm}^{-2} \mathrm{~s}^{-1} \AA^{-1}$, with $\mathrm{n}=0,1 \ldots$. The velocity scale is centered on the laboratory wavelength of C IV $\lambda 1548$ and corrected for an SMC $+150 \mathrm{~km} \mathrm{~s}^{-1}$ systemic velocity. 

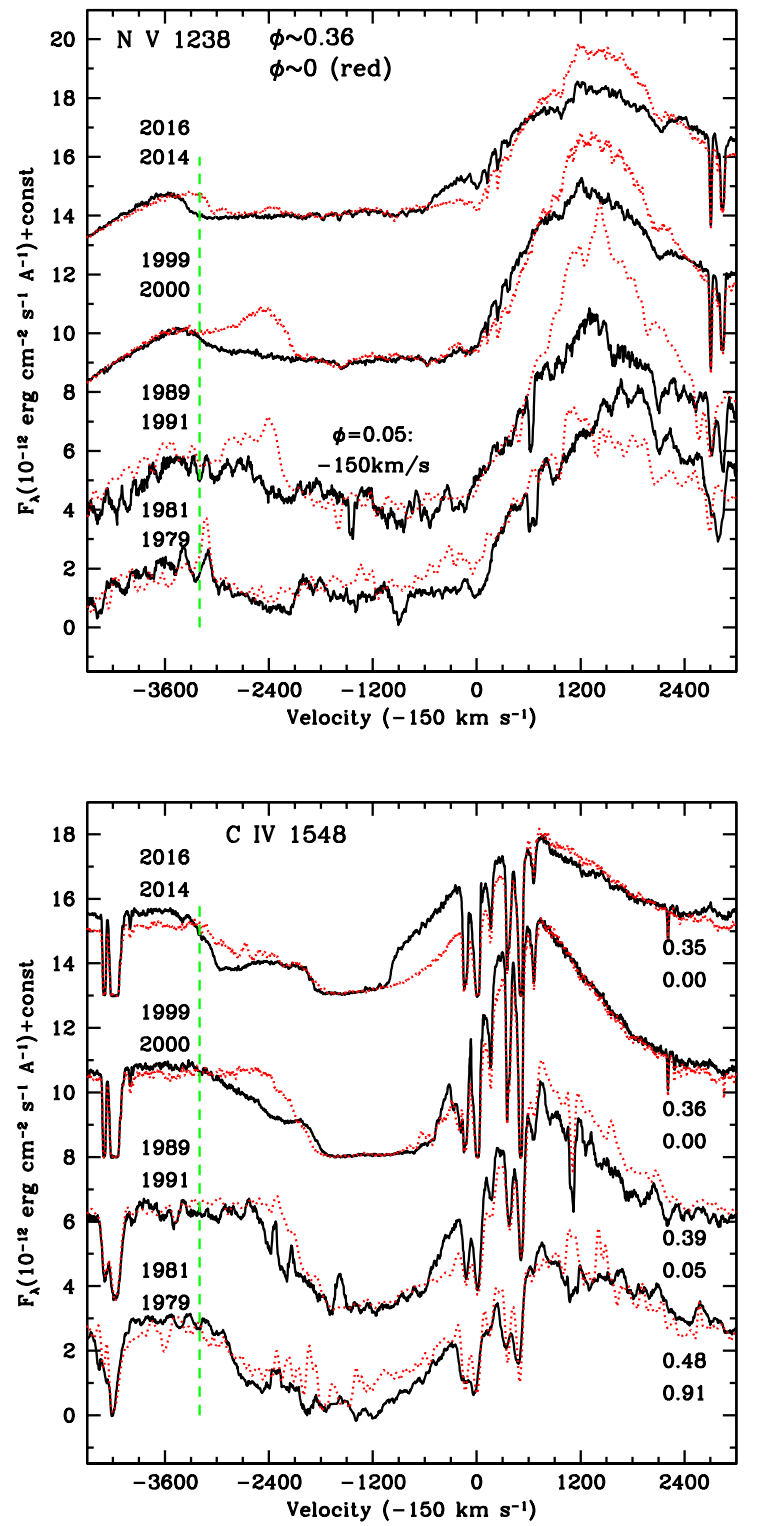

Figure 19. Line profiles at orbital phase $\sim 0.36$ at different epochs compared with their counterparts around $\phi \sim 0.0$ (dotted, red). Years (shown at left in each panel) and phases (shown at right in each panel) are given for $\phi \sim 0.36$ in the upper listing and for $\phi \sim 0.0$ in the lower listing. The $\mathrm{N} \mathrm{V}$ profile at $\phi=0.05$ was shifted by $-150 \mathrm{~km} \mathrm{~s}^{-1}$ to correct for the orbital motion of Star A at that phase. This same shift was not applied to the C IV profile.

show the C IV $\lambda \lambda 1548,1552$ profile at both eclipses. The extended absorption blueward of $V_{\text {black }}$ is easily seen. In a steady wind with a monotonic velocity law we expect the blue edge to be sharp and vertical, since the optical depth will still (generally) be large when the wind reaches its terminal speed. For the 2014 data set it might be possible to use the same explanation for the extended absorption on C IV $\lambda \lambda 1548,1552$, however the absorption on $\mathrm{N}$ V $\lambda \lambda 1238,1242$ is almost black to $3000 \mathrm{~km} \mathrm{~s}^{-1}$ suggesting this is unlikely to be the explanation. Further, the presence of the same phenomenon at other epochs, even when Star A's terminal
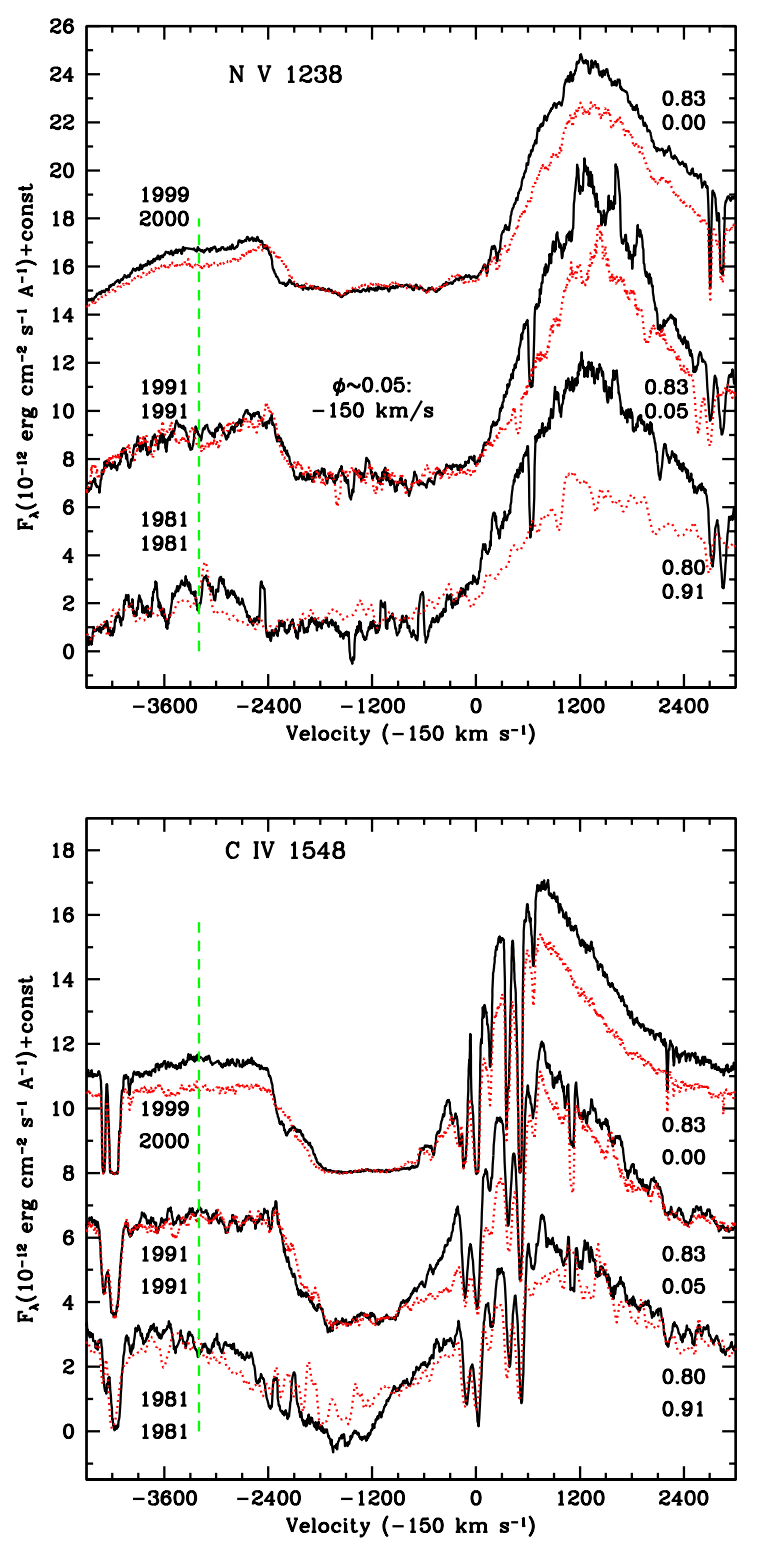

Figure 20. Line profiles at orbital phase $\sim 0.80$ at different epochs compared with their counterparts around $\phi \sim 0.0$ (dotted, red). Years (shown at left in each panel) and phases (shown at right in each panel) are given for $\phi \sim 0.8$ in the upper listing and for $\phi \sim 0.0$ in the lower listing.

velocity was much lower, would suggest that the extended absorption is associated with the binary nature of the system.

(ii) In 2014, $V_{\infty}^{A}=-3000 \mathrm{~km} \mathrm{~s}^{-1}$ and the neglect in CMFGEN of the binary interaction effects leads to the discrepancy between the model fit and the observed spectrum. Since both components of the AB binary possess strong winds, a wind-wind interaction region necessarily is present. As noted earlier, the semi-major axis of the system is only $\sim 7$ stellar radii. Thus when Star A is eclipsing Star B, its wind on the far side from the observer will be substantially modified. Second, the similarity of the stars makes it likely that the radiation from each of them is strongly affecting the wind 
in the hemisphere facing its companion. In the simplest scenario (and assuming emission from Star $A$ dominates) this would give rise to asymmetric and blue-shifted emission profiles, however this is not obvious in the 2014 data.

(iii) Star $B$ has a fast wind with $V_{\infty}^{B} \sim 3000 \mathrm{~km} \mathrm{~s}^{-1}$. As Star $A$ passes in front of Star B, we see the former wind of Star $B$ in projection against Star A, producing the anomalous absorption. As noted earlier, the anomalous absorption has been becoming more evident as Star A's mass loss declines. Two effects can be contributing to this evolution. First, as the mass loss declines the opening angle of the bow shock will increase, allowing a more prominent Star B wind. However, calculations by Nazé et al. (2018) show that this is not a large effect, at least after 2000. Second, when Star A was in its "bright" state, its thicker wind could have prevented UV photons from maintaining the ionization of Star B's wind.

(iv) The wind of Star $A$ is modified by the radiation field of Star B. This idea is supported by the study of FUSE spectra (Koenigsberger et al. 2006), in which the wind structure of Star A in the direction of Star B was found to be altered with respect to other orientations. Usually we are primarily concerned with the influence of the radiation field altering the wind structure between the stars. However, it can also potentially affect the freely streaming wind. For example, if we assume that the two stars are identical, the flux from Star $B$ at $10 R_{*}$ from Star $A\left(\sim 17.5 R_{*}\right.$ from Star B, and ignoring occultation effects) is $\sim 30 \%$ of Star $A$ which is significant enough to enhance the acceleration of Star A's wind, especially if its flux distribution is better tuned to drive the wind of Star A (i.e., if the peak of its flux distribution better matches the line distribution). The extended absorption is produced in wind regions that lie beyond the orbital radius and are subjected to a velocity "boost" from the emerging radiation field of both stars.

(v) The wind of Star A (and/or Star B) is intrinsically aspherical and/or its structure is variable on orbital timescales. The orbit is sufficiently eccentric so that the tidal interaction leads to a more bloated radius around periastron than around apastron. Assuming Star $A$ has $\sim 24 R_{\odot}$, the equatorial maximum radius is $\sim 0.05 R_{\odot}$ larger at periastron than at apastron. Although this may seem like an insignificant effect, the potentially non-linear interplay between radius, luminosity, mass-loss rate and wind speed could lead to a transition in the wind structure between periastron and apastron. In addition, the incoming radiation field from Star $B$ also varies between periastron and apastron, being more diluted at apastron.

(vi) A final possibility is the outward spiraling of the colliding wind region, as is detected in some WR systems. As mentioned below, we need UV spectra out of eclipses to see if the extended component is present.

Some of these alternatives could be tested with new observations over an entire orbital cycle, as with those obtained by IUE in 1989-91 and HST in 1999. We illustrate in Fig. 21 pairs of $\mathrm{N} \mathrm{V} \lambda \lambda 1238,1242$ line profiles obtained in 1991 and 1999 at the same two orbital phases. The UV spectra at these two epochs are similar, although the latter epoch has stronger lines and a higher continuum level. Despite these epoch-related differences, the phase-dependent variations present in Fig. 21 are the same, within the uncer-

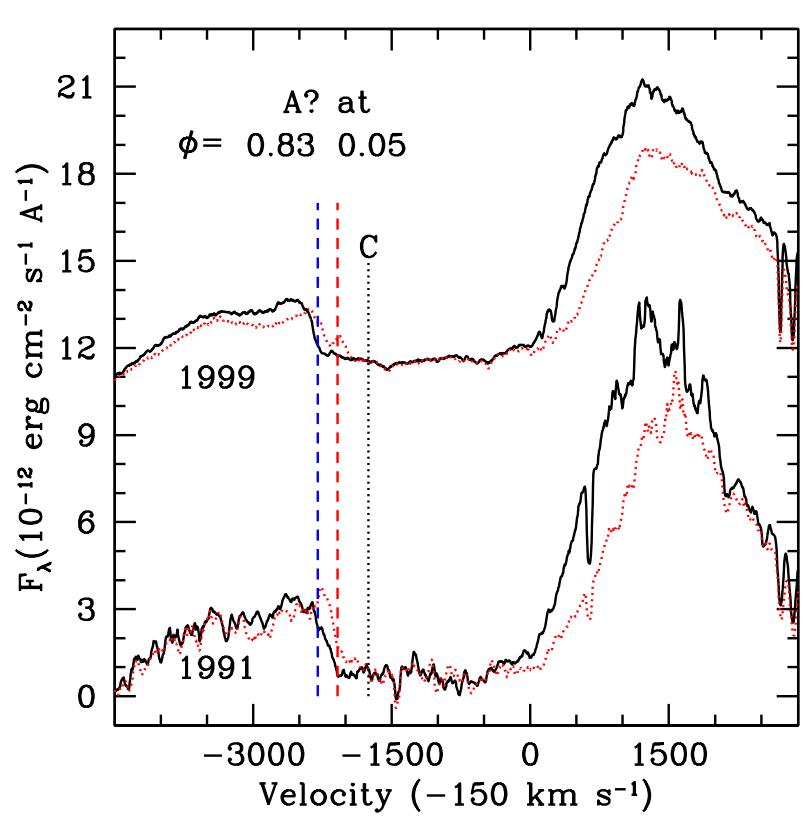

Figure 21. Comparison of 1999 and 1991 line profiles of N V at the same two orbital phases, 0.05 (dotted, red) and 0.83 (black). No horizontal shifts to correct for orbital motion have been applied. Dashed lines indicate expected location of the Star A P Cygni absorption edge at the two orbital phases, assuming it is located at $2200 \mathrm{~km} \mathrm{~s}^{-1}$ in the Star $A$ rest frame. The 1999 pair of spectra is shifted vertically for clarity in the figure.

tainties of the data. Specifically, there is a clear velocity shift between orbital phase $\phi=0.83$, when $\operatorname{Star} A$ is approaching the observer and $\phi=0.05$, when it is receding, that is consistent with the orbital motion of Star A. The phase-related difference in the emission component is also the same. In summary, it may now be possible to pin down the nature of the interaction effects causing phase-dependent variations as observed in HD 5980 which, once understood, may shed light on peculiarities that are emerging from the analysis of other, more distant and less well studied massive star systems.

\subsection{The 2016 data - eclipse of Star A by Star B}

As discussed earlier, the 2016 data show unusual profiles. First, many of the emission profiles are asymmetric. This effect is easily seen in the UV data, and is even evident in the lower resolution optical data (Fig. 12). Second, the C IV $\lambda \lambda 1548,1552$ and N V $\lambda \lambda 1238,1242 \mathrm{P}$ Cygni absorption components show an anomalous structure. Third, the C IV $\lambda \lambda 1548,1552$ transition, unlike $\mathrm{N} \mathrm{V} \lambda \lambda 1238,1242$ shows a blue-shifted profile. What is the cause of the unusual profiles?

The asymmetry of He II $\lambda 1640$ suggests an absence of red-shifted emission rather than an enhancement of blueshifted emission. This can be seen in Fig. 12, where we compare the 2014 and 2016 UV data. Conversely, the C IV $\lambda \lambda 1548,1552$ profile exhibits enhanced blue emission, as well as an apparent suppression of the emission on the red side (Figs. 11 \& 22). However we do not see any obvious indication of red shifted absorption. At phase 0 , such absorption 

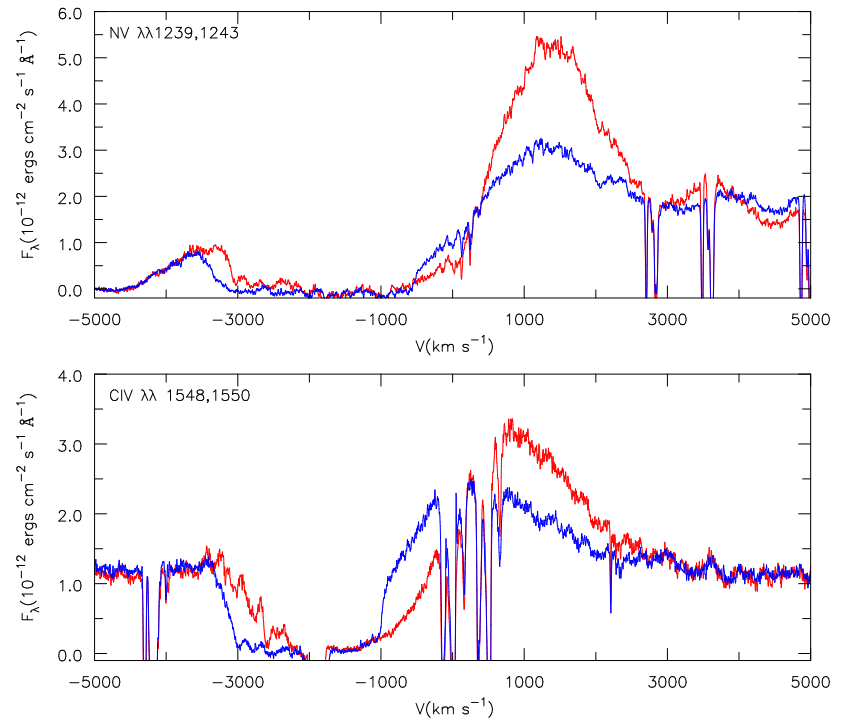

Figure 22. Comparison between the N V $\lambda \lambda 1238,1242$ and C IV $\lambda \lambda 1548,1552$ resonance profiles obtained in 2014 (phase 0.0 , red) and 2016 (phase 0.37, blue). For display purposes the 2016 UV spectrum has been divided by 1.3 .

could arise as photons from Star A pass through the receding hemisphere (as viewed from Earth) of Star B's wind.

One possible interpretation of the 2016 data is that the emission lines are primarily coming from Star B. The asymmetry is caused by the interaction of Star A's wind with Star B's wind which suppresses some of the red emission coming from Star B. The same mechanism can explain the blue shifted C IV $\lambda \lambda 1548,1552$ emission and the lack of blue shift for N V $\lambda \lambda 1238,1242$. For the N V doublet the emission component is formed deeper in the wind, and hence will not be so strongly influenced by the modifications of Star A's wind by Star $B$.

What about the sudden onset of extra absorption in the C IV $\lambda \lambda 1548,1552$ and N V $\lambda \lambda 1238,1242$ profiles? After making allowances for the doublet structure of the resonance transitions, the data suggest that the absorption begins at a velocity of $1500 \mathrm{~km} \mathrm{~s}^{-1}$. As the absorption is contaminated by the classical P Cygni absorption the maximum velocity of this gas can only be constrained if we assume it dominates the absorption component. In this case the two resonance lines indicate velocities approaching $\sim 3500 \mathrm{~km} \mathrm{~s}^{-1}$, with the maximum extent of the "black" absorption trough indicating velocities of $\sim 3000 \mathrm{~km} \mathrm{~s}^{-1}$. Since the intensity in the trough goes to zero, the absorbing gas must be located between us and both stars, that is, on the near side of Star B.

The "detached" absorption could be caused by a partial shell of material that absorbs light from both Star $A$ and Star B. Such a shell could be produced, for example, if the inner region of Star B's wind was so highly ionized that no C IV $\lambda \lambda 1548,1552$ absorption is produced for velocities less than $1500 \mathrm{~km} \mathrm{~s}^{-1}$. By itself, this scenario cannot explain the blue-shifted C IV $\lambda \lambda 1548,1552$ emission.

\section{CONCLUSIONS}

The observations of HD 5980 obtained by HST/STIS in 2014 indicate that the mass-loss rate and luminosity of Star $A$ have reached the lowest values ever determined: $\dot{M} \sim 4.5 \times 10^{-5}, L \sim 1.7 \times 10^{6} L_{\odot}$. In addition, the best-fit radius of the continuum-emitting region is similar to that derived from the eclipse light curves of the late 1970s. Hence, it appears to have attained a similar "low" state to that of the late 1970s. The present estimates of the parameters of Star $A$ were derived assuming that the spectrum at phase 0 is dominated by Star A. However as Star A has faded, and as its mass loss shrinks, this assumption is becoming less tenable. Thus it is probable that the values given above are overestimates for this star.

Despite the good fit to the emission spectrum taken in 2014 at phase 0, there is a discrepancy between the observed P Cygni absorption components of the resonance lines and the model fits. Specifically, the observed profiles are extended by more than $\sim 1000 \mathrm{~km} \mathrm{~s}^{-1}$ over that predicted by the emission line widths. Assuming a larger terminal wind speed for the model does not resolve this issue, since it results in emission-line profiles that are too strong compared to the observations. Hence, the explanation may lie in a departure from spherical symmetry in the wind of the star being modeled, an effect not accounted for by the CMFGEN models. The asymmetry is likely to be related to the perturbation of the LBV/WR wind by radiation from the companion star, and the wind-wind interaction. It is noteworthy that the discrepancy between the CMFGEN models and the observations has become greater as the mass-loss rate has been declining.

The UV data taken in 2016 provides an even larger puzzle. In particular, both $\mathrm{N} \mathrm{V} \lambda \lambda 1238,1242$ and $\mathrm{C} \mathrm{IV}$ $\lambda \lambda 1548,1552$ show detached absorption in their $\mathrm{P}$ Cygni absorption components. From the "detached" absorption we infer the existence of material with velocities from 1500 to $3000 \mathrm{~km} \mathrm{~s}^{-1}$ (and possibly as high as $3500 \mathrm{~km} \mathrm{~s}^{-1}$ ) along our line of sight. Another puzzle is that emission lines are asymmetric. The He II $\lambda 1640$ profile indicates a lack of red-shifted emission, while the C IV $\lambda \lambda 1548,1552$ doublet profile is noticeably shifted to the blue. The anomalous profiles are not seen in data taken in 1999 at the same phase, and are not readily apparent in any other data sets.

Detailed hydrodynamical modeling is required in order to constrain the wind-wind interaction region, as is a multidimensional treatment of the radiative transfer. The relatively short period and strong winds means dynamical effects are important - at any phase we cannot assume that we are looking at a system which has reached equilibrium. This is seen in the data - profiles at the two greatest elongations (phases $\sim 0.14$ and $\sim 0.76$ ) are inconsistent with each other (e.g., Foellmi et al. 2008), and this is unlikely to be due to the difference in orbital separation. Further, modification of the winds by the radiation field of the companion star will occur, and because of the elliptical orbit, will be phase dependent.

It is important to note that the type of interaction effects we describe here are expected to be prevalent among massive binary systems in general, and that the proper fitting of their spectra will also require these interaction effects to be taken into account. The only difference between the many expected systems with these effects and HD 5980 is 
that in the latter the effects are accentuated, due to the large mass-loss rate, which allows them to be studied more easily. HD 5980 also provides the extraordinary opportunity for modeling a system in which different momentum balances have been present at different times, but for which the masses and orbital parameters have remained relatively constant. Because the system is eclipsing, because of similarities in the star masses and luminosities, and because of the variability of Star A, current and future observations of this system will provide strong constraints on binary interactions, interacting winds, and radiation damping.

Acknowledgements: Data presented in this paper were obtained from the Mikulski Archive for Space Telescopes (MAST). STScI is operated by the Association of Universities for Research in Astronomy, Inc., under NASA contract NAS 5-26555. Support for MAST for non-HST data is provided by the NASA Office of Space Science via grant NNX09AF08G and by other grants and contracts. Support to DJH for this work was provided by NASA through grant numbers HST-GO-13373.001-A and HST-GO-14476.002-A from the Space Telescope Science Institute, which is operated by AURA, Inc., under NASA contract NAS 5-26555. GK acknowledges support from CONACYT 252499 and DGAPA/UNAM IN103619. YN acknowledges support from the Fonds National de la Recherche Scientifique (Belgium), and the PRODEX XMas contract. RHB acknowledges support from DIDULS Project PR18143 (Universidad de La Serena, Chile) We would to like to express our gratitude to the referee, Ian Howarth, for a thorough and comprehensive review of our paper. Any mistakes remaining in the paper are the sole responsibility of the authors.

\section{REFERENCES}

Abbott B. P., et al., 2016, Phys. Rev. D, 93, 122003

Azzopardi M., Vigneau J., 1975, A\&AS, 19, 271

Barbá R. H., Niemela V. S., Baume G., Vazquez R. A., 1995, ApJ, 446, L23+

Barbá R., et al., 1996, in Falco E., Fernandez J. A., Ferrero R. F., eds, Revista Mexicana de Astronomia y Astrofisica Conference Series Vol. 4, Revista Mexicana de Astronomia y Astrofisica Conference Series. p. 90

Bateson F. M., Jones A. F., 1994, Royal Astronomical Society of New Zealand Publications of Variable Star Section, 19, 50

Breysacher J., 1997, in Nota A., Lamers H., eds, Astronomical Society of the Pacific Conference Series Vol. 120, Luminous Blue Variables: Massive Stars in Transition. p. 227

Breysacher J., Perrier C., 1980, A\&A, 90, 207

Breysacher J., Perrier C., 1991, in van der Hucht K. A., Hidayat B., eds, IAU Symposium Vol. 143, Wolf-Rayet Stars and Interrelations with Other Massive Stars in Galaxies. p. 229

Breysacher J., Westerlund B. E., 1978, A\&A, 67, 261

Cardelli J. A., Clayton G. C., Mathis J. S., 1989, ApJ, 345, 245

Drissen L., Crowther P. A., Smith L. J., Robert C., Roy J.-R., Hillier D. J., 2001, ApJ, 546, 484

Feast M. W., Thackeray A. D., Wesselink A. J., 1960, MNRAS, 121,337

Foellmi C., et al., 2008, Rev. Mex. Astron. Astrofis., 44, 3

Gayley K. G., Owocki S. P., Cranmer S. R., 1996, in Niemela V., Morrell N., Pismis P., Torres-Peimbert S., eds, Revista Mexicana de Astronomia y Astrofisica, vol. 27 Vol. 5, Revista Mexicana de Astronomia y Astrofisica Conference Series. p. 68
Georgiev L., Koenigsberger G., Hillier D. J., Morrell N., Barbá R., Gamen R., 2011, AJ, 142, 191

González R. F., Koenigsberger G., 2014, A\&A, 561, A105

Gräfener G., Hamann W.-R., 2005, A\&A, 432, 633

Hainich R., Pasemann D., Todt H., Shenar T., Sander A., Hamann W.-R., 2015, A\&A, 581, A21

Heydari-Malayeri M., Rauw G., Esslinger O., Beuzit J.-L., 1997, A\&A, 322, 554

Hilditch R. W., Howarth I. D., Harries T. J., 2005, MNRAS, 357,304

Hillier D. J., Miller D. L., 1998, ApJ, 496, 407

Koenigsberger G., 2004, Rev. Mex. Astron. Astrofis., 40, 107

Koenigsberger G., Moffat A. F. J., St-Louis N., Auer L. H., Drissen L., Seggewiss W., 1994, ApJ, 436, 301

Koenigsberger G., Shore S., Guinan E., Auer L., 1996, in Niemela V., Morrell N., Pismis P., Torres-Peimbert S., eds, Revista Mexicana de Astronomia y Astrofisica Conference Series Vol. 5, Revista Mexicana de Astronomia y Astrofisica Conference Series. p. 92

Koenigsberger G., Auer L. H., Georgiev L., Guinan E., 1998a, ApJ, 496, 934

Koenigsberger G., Peña M., Schmutz W., Ayala S., 1998b, ApJ, 499, 889

Koenigsberger G., Kurucz R. L., Georgiev L., 2002, ApJ, 581, 598

Koenigsberger G., Fullerton A. W., Massa D., Auer L. H., 2006, AJ, 132,1527

Koenigsberger G., Georgiev L., Hillier D. J., Morrell N., Barbá R., Gamen R., 2010, AJ, 139, 2600

Koenigsberger G., Morrell N., Hillier D. J., Gamen R., Schneider F. R. N., González-Jiménez N., Langer N., Barbá R., 2014, AJ, 148,62

Mardling R., Aarseth S., 1999. p. 385

Moffat A. F. J., et al., 1998, ApJ, 497, 896

Nazé Y., Koenigsberger G., Pittard J. M., Parkin E. R., Rauw G., Corcoran M. F., Hillier D. J., 2018, ApJ, 853, 164

Neugent K. F., Massey P., Hillier D. J., Morrell N., 2017, ApJ, 841,20

Niemela V. S., 1988, in Blanco V. M., Phillips M. M., eds, Astronomical Society of the Pacific Conference Series Vol. 1, Progress and Opportunities in Southern Hemisphere Optical Astronomy. The CTIO 25th Anniversary Symposium. p. 381

Niemela V. S., Barbá R. H., Morrell N. I., Corti M., 1997, in Nota A., Lamers H., eds, Astronomical Society of the Pacific Conference Series Vol. 120, Luminous Blue Variables: Massive Stars in Transition. p. 222

Pablo H., et al., 2017, MNRAS, 467, 2494

Perrier C., Breysacher J., Rauw G., 2009, A\&A, 503, 963

Richardson N. D., et al., 2018, MNRAS, 475, 5417

Schmutz W., 1997, A\&A, 321, 268

Schmutz W., Vacca W. D., 1991, A\&AS, 89, 259

Schweickhardt J., 2000, PhD thesis, PhD Thesis, Landessternwarte Heidelberg/Königstuhl

Schweickhardt J., Wolf B., Schmid H. M., Kaufer A., Stahl O., Szeifert T., Tubbesing S., 2000, in Lamers H., Sapar A., eds, Astronomical Society of the Pacific Conference Series Vol. 204, Thermal and Ionization Aspects of Flows from Hot Stars. p. 113

Scowcroft V., Freedman W. L., Madore B. F., Monson A., Persson S. E., Rich J., Seibert M., Rigby J. R., 2016, ApJ, 816, 49

Shenar T., et al., 2016, A\&A, 591, A22

Sterken C., Breysacher J., 1997, A\&A, 328, 269

Stevens I. R., Blondin J. M., Pollock A. M. T., 1992, ApJ, 386, 265

Toonen S., Hamers A., Portegies Zwart S., 2016 , Computational Astrophysics and Cosmology, 3, 6

Walborn N. R., 1977, ApJ, 215, 53 